\title{
Neutrino interaction measurements with the MicroBooNE and ArgoNeuT liquid argon time projection chambers
}

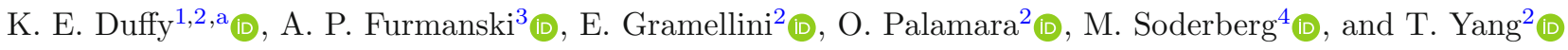 \\ 1 University of Oxford, Oxford OX1 3RH, UK \\ ${ }^{2}$ Fermi National Accelerator Laboratory (FNAL), Batavia, IL 60510, USA \\ 3 University of Minnesota, Minneapolis, MN 55455, USA \\ 4 Syracuse University, Syracuse, NY 13244, USA
}

Received 18 June 2021 / Accepted 23 September 2021 / Published online 11 January 2022

(C) The Author(s) 2022

\begin{abstract}
Precise modeling of neutrino interactions on argon is crucial for the success of future experiments such as the Deep Underground Neutrino Experiment (DUNE) and the Short-Baseline Neutrino (SBN) program, which will use liquid argon time projection chamber (LArTPC) technology. Argon is a large nucleus, and nuclear effects - both on the initial and final-state particles in the interaction - are expected to be large in neutrino-argon interactions. Therefore, measurements of neutrino scattering cross sections on argon will be of particular importance to future DUNE and SBN oscillation measurements. This article presents a review of neutrino-argon interaction measurements from the MicroBooNE and ArgoNeuT collaborations, using two LArTPC detectors that have collected data in the NuMI and Booster Neutrino Beams at Fermilab. Measurements are presented of charged-current muon neutrino scattering in the inclusive channel, the ' $0 \pi$ ' channel (in which no pions but some number of protons may be produced), and single pion production (including production of both charged and neutral pions). Measurements of electron neutrino scattering are presented in the form of $\nu_{e}+\bar{\nu}_{e}$ inclusive scattering cross sections.
\end{abstract}

\section{Introduction}

One of the largest challenges faced by accelerator-based neutrino oscillation experiments is understanding the interactions of neutrinos with the target nucleus constituting the detectors. Neutrino-nucleus interactions are inherently challenging to model, and experiments must rely on data to tune models for use in oscillation analyses $[1,2]$. Even for a simple interaction, such as quasielastic scattering off a single nucleon in a nucleus, models must correctly consider the initial state motion and binding energy of that nucleon, as well as its interactions with the residual nucleus as it makes its way from an interaction point out of the nucleus (these are known as final-state interactions, or FSIs). Recent electron scattering measurements have shown the importance of correlations between nucleons inside a nucleus, further complicating the picture. For these reasons, many models have historically relied heavily on data [2]. Ab initio calculations that consider all nuclear effects are in development (e.g. [3]), which will reduce reliance on data, but nevertheless it is important that these calculations are compared to data.

\footnotetext{
a e-mail: kirsty.duffy@physics.ox.ac.uk (corresponding author)
}

A significant amount of neutrino interaction measurements have been made with carbon targets over the past decades, as well as some measurements on other similar sized nuclei; generators and models have been tuned to this data to sufficient precision for current oscillation measurements. However, for the future Deep Underground Neutrino Experiment (DUNE) [4] and the Short Baseline Neutrino (SBN) program [5] it is critical that our understanding of neutrino-nucleus interactions is extended to argon targets, since both will use liquid argon technology as the primary neutrino detection method. As argon is a significantly larger nucleus than carbon, nuclear effects generally have a larger impact on the kinematics of initial and final state particles so even where models can successfully describe data on carbon, it is important to verify that they scale to argon correctly. In addition to this, future oscillation measurements will require further reduced neutrino interaction uncertainties, requiring more precise measurements on argon than exist for carbon.

This article presents a review of neutrino interaction measurements by two experiments-ArgoNeuT and MicroBooNE - which together form the majority of all neutrino-argon interaction measurements globally. The energy ranges of these measurements are of particular relevance to DUNE and SBN: the ArgoNeuT measurements sample the primary DUNE oscillation 
energy; and MicroBooNE measurements are close to the energy of the second oscillation maximum at DUNE, where $\mathrm{CP}$-violation effects are larger, as well as being an identical energy range to the SBN experiments.

Due to the need for higher precision, modeling of neutrino-nucleus interactions has been developed considerably over the past decade. Therefore the models used for developing analyses, as well as the models compared to, have some differences throughout this article. Both the experiments discussed used the GENIE monte carlo $(\mathrm{MC})$ generator $[6,7]$ as their primary simulation. The ArgoNeuT experiment used GENIE v2.6 and GENIE v2.8 for most analyses. The majority of MicroBooNE analyses use GENIE v2.12 as the primary simulation. The largest difference between these versions is the addition of meson-exchange current (MEC) interactions (also known as 2p2h or multinucleon interactions) in v2.12. Data are additionally compared to GENIE v3.0 [8] predictions, which include a number of theoretical updates, as well as NuWro [9], GiBUU [10], and NEUT $[1,11]$. Care must be taken when interpreting comparisons of data to predictions, as the newer data are generally compared to newer simulations.

In addition to being the first measurements of various interaction types with an argon target, the use of liquid argon TPC technology means these measurements take advantage of the full 3D imaging, precise tracking, calorimetric energy reconstruction, particle identification, and low energy thresholds available. This has led to a number of measurements of exclusive topologies with detailed characterization of the full final state, leading to more stringent tests of models.

\section{LArTPCs in action: ArgoNeuT and MicroBooNE}

A comprehensive introduction to liquid argon time projection chamber (LArTPC) detector technology may be found in $[12,13]$. Several features of this technology make these detectors a versatile tool for the study of neutrino interactions; LArTPCs are effectively fullyactive tracking calorimeters. They bestow tridimensional imaging, tracking, calorimetry, and - in many cases - a self-triggering mechanism provided by scintillation light in liquid argon. Leveraging both the highresolution tracking and calorimetry, excellent particle identification capabilities have been achieved in LArT$\mathrm{PCs}$ over a large kinematic phase space.

This article presents a review of neutrino interaction measurements in two LArTPC detectors: ArgoNeuT [14] and MicroBooNE [15]. ArgoNeuT was the first LArTPC detector in a low-energy neutrino beam: it was designed as a test experiment, but obtained physics results. ArgoNeuT's physics run lasted from September 2009 through February 2010, and featured a $\sim 0.25$ ton active volume LArTPC, with dimensions of $47.5 \mathrm{~cm}$ (drift) $\times 40.0 \mathrm{~cm}$ (height) $\times 90.0 \mathrm{~cm}$ (length) exposed to Fermilab's Neutrinos from the Main Injector (NuMI) beamline [16]. The TPC had three anode planes, all with $4 \mathrm{~mm}$ wire pitch, with two planes instrumented for electronic readout. ArgoNeuT did not have a light collection system, and instead relied upon timing signals from the accelerator complex to trigger on NuMI beam spills. The detector was located underground in the NuMI tunnel at Fermilab, immediately upstream of the MINOS Near Detector (MINOS-ND) [17] which served as a spectrometer for muons exiting ArgoNeuT. The magnetized MINOS-ND detector allowed ArgoNeuT to make measurements separating muon neutrino and antineutrino interactions, and the underground location means that the cosmic background was negligible. Sitting on-axis with respect to the NuMI beamline, a data sample of $1.335 \times 10^{20}$ protons on target (POT) was collected in the "low-energy" NuMI configuration, composed of $8.5 \times 10^{18} \mathrm{POT}$ in neutrino mode $\left(\left\langle E_{\nu}\right\rangle=4.3 \mathrm{GeV}\right)$ and $1.25 \times 10^{20}$ POT in antineutrino mode $\left(\left\langle E_{\nu}\right\rangle=9.6 \mathrm{GeV},\left\langle E_{\bar{\nu}}\right\rangle=3.6 \mathrm{GeV}\right)$.

MicroBooNE [15] is the first detector of the SBN program to be fully operational, starting its first neutrino data-taking run in October 2015. The SBN program is comprised of three surface LArTPCs positioned on-axis in Fermilab's Booster Neutrino Beam (BNB) [18]: SBND (110 $\mathrm{m}$ from the decay pipe), MicroBooNE $(470 \mathrm{~m})$, and ICARUS $(600 \mathrm{~m})$ [5]. MicroBooNE is an 85 ton active volume LArTPC with TPC dimensions of $2.6 \mathrm{~m}$ (drift) $\times 2.3 \mathrm{~m}$ (height) $\times 10.4 \mathrm{~m}$ (length) . The TPC has three anode planes, with $3 \mathrm{~mm}$ wire pitch, all instrumented for electronic readout (a total of 8256 wires in the detector readout). MicroBooNEs light collection system consists of 32 photomultiplier tubes (PMTs), each of $200 \mathrm{~mm}$ diameter, which register scintillation light produced in neutrino interactions to provide the start time for electron drift (allowing three-dimensional track reconstruction). Optical signals are also used to discriminate between TPC activity caused by neutrino interactions and cosmic rays. Because MicroBooNE sits on the surface, cosmic ray backgrounds are significant; the impact of these events is estimated using data collected when the beam is not running. In December 2017, a cosmic ray tagger [19] was installed, although the measurements presented in this article use only data collected prior to that date. The on-axis BNB neutrino flux has an average energy of $\sim 800 \mathrm{MeV}$, and is used for the measurements of muon neutrino interactions presented in this article. MicroBooNE also detects neutrinos produced by Fermilab's NuMI beam [16]. The MicroBooNE detector is situated close to $8^{\circ}$ off axis with respect to the NuMI beamline, leading to a neutrino flux in the "medium energy" neutrino-mode configuration with a mean energy of $905 \mathrm{MeV}$ and a composition of roughly $96 \% \nu_{\mu}+\bar{\nu}_{\mu}$ and $4 \% \nu_{e}+\bar{\nu}_{e}[20]$. This enables high-statistics measurements of electron neutrino interactions. To date, MicroBooNE has collected an exposure of $1.52 \times 10^{21}$ POT in the BNB flux and $2.33 \times 10^{21}$ POT in NuMI, although only subsamples of these data sets have currently been analysed (exposures for individual measurements are given in the following sections). 


\section{Measurements of $\nu_{\mu}$ and $\bar{\nu}_{\mu}$ charged-current inclusive scattering}

A charged-current (CC) inclusive event selection can be relatively simple, if it requires only the outgoing muon ( $\mu^{+}$in the case of $\bar{\nu}_{\mu}$ interactions) to be reconstructed. These event selections allow for overall tests of neutrino interaction models, as well as forming possible foundations for studies of more complex event topologies (for example, the MicroBooNE $\nu_{\mu}$ CC inclusive selection in Sect. 3.2 is used as a preselection for the $\nu_{\mu} \mathrm{CC} 0 \pi \mathrm{Np}$ measurement in Sect. 4.2.1). In terms of interaction modes, the inclusive signal definition includes many different types of neutrino interaction, with the exact mixture depending on the composition and energy of the neutrino beam flux being studied. The on-axis BNB neutrino flux at MicroBooNE is relatively low-energy, with a mean neutrino energy of $0.8 \mathrm{GeV}$. Therefore, the dominant interaction processes in MicroBooNE's $\mathrm{CC}$ inclusive measurement will be quasielastic (QE) and $2 \mathrm{p} 2 \mathrm{~h}$ scattering. ArgoNeuT, on the other hand, uses the on-axis NuMI low-energy beam, with a mean neutrino energy of $4.3 \mathrm{GeV}\left(\nu_{\mu}\right.$ in the neutrino-mode beam), 9.6 GeV ( $\nu_{\mu}$ in the antineutrino-mode beam), or $3.6 \mathrm{GeV}\left(\bar{\nu}_{\mu}\right.$ in the antineutrino-mode beam). Due to this higher neutrino energy, ArgoNeuT expects to see significant contributions from neutrino-induced resonance production (RES) and deep inelastic scattering (DIS) in the CC inclusive selection, in addition to quasielastic and $2 \mathrm{p} 2 \mathrm{~h}$ scattering.

\subsection{ArgoNeuT $\nu_{\mu}$ and $\bar{\nu}_{\mu} \quad \mathrm{CC}$ inclusive cross-section measurement}

References $[21,22]$ report the first measurements of the inclusive muon neutrino and antineutrino charged current cross sections on argon using the ArgoNeuT detector in the NuMI beamline.

The initial muon neutrino measurement was made using a data sample of $8.5 \times 10^{18}$ POT, collected during a very brief $\sim 2$ week run of ArgoNeuT when the NuMI beam was in the low-energy neutrino-mode configuration. Event candidates are required to have a candidate neutrino interaction vertex inside of a fiducial volume $\left(3 \mathrm{~cm}<x_{v t x}<44 \mathrm{~cm},-16 \mathrm{~cm}<y_{v t x}<16 \mathrm{~cm}, 6 \mathrm{~cm}<\right.$ $z_{v t x}<86 \mathrm{~cm}$ ), with one reconstructed track originating from the vertex matched to a negatively-charged track starting within the first $20 \mathrm{~cm}$ of the MINOS-ND. Figure 1 shows the flux-integrated differential CC inclusive cross section measured in this analysis as a function of reconstructed muon momentum and scattering angle, compared against a prediction made using GENIE v2.6. In general, good agreement with the model was found, except potentially in the lowest bins of both muon momentum and scattering angle. The small statistical size of the data sample makes it difficult to draw further conclusions.

A subsequent measurement of both muon neutrino and antineutrino CC inclusive cross sections was made using a larger $1.25 \times 10^{20}$ POT data sample recorded by ArgoNeuT over several months when the NuMI beam was in the low-energy antineutrino-mode configuration. The sizeable neutrino component of the antineutrino-mode beam, combined with the capability of the MINOS-ND to perform sign selection of muons emanating from ArgoNeuT, enabled this comprehensive suite of measurements. The same fiducial volume definition and muon matching requirements were utilized in this analysis, with the addition that both negatively and positively charged MINOS-ND tracks were allowed, to differentiate between neutrino and antineutrino interactions respectively. Figure 2 shows the measured flux-integrated differential CC inclusive cross section for neutrinos (Left) and antineutrinos (Right) as a function of reconstructed muon momentum (Bottom) and scattering angle (Top), compared against predictions made using GENIE v2.8.0 and NUWRO version $11 \mathrm{~m}$ (with a spectral function and "TE" meson exchange currents). As in the case of the neutrinomode analysis described above, though this time with larger statistics, generally good agreement is observed between the measurement and the models across the momentum and scattering angle distributions.

\subsection{MicroBooNE $\nu_{\mu}$ CC inclusive cross-section measurement}

Reference [23] reports the first measurement of the double-differential (in muon momentum and scattering angle) muon neutrino charged current inclusive cross section on argon, using the MicroBooNE detector. This measurement uses an exposure of $1.6 \times 10^{20}$ POT from the on-axis BNB, and selects 27,200 candidate events with an estimated $\nu_{\mu} \mathrm{CC}$ interaction purity of $50.4 \%$; this corresponds to the largest sample of neutrino interactions on argon published to date. The main background to this measurement (29.1\% of all selected events) comes from cosmic rays, and is estimated using data.

The initial stage of the selection consists of cuts to remove backgrounds from cosmic rays using the timing of the interaction, correlations between optical and TPC information, and event topology. Muons are then identified using calorimetric information in the form of a truncated mean of the deposited charge per unit length $(\mathrm{d} Q / \mathrm{d} x)$ and track length, to reject neutralcurrent interactions. The muon momentum is reconstructed using multiple Coulomb scattering [24], which allows a measurement of muon momentum for muons either contained in, or exiting the detector. This means that this measurement is able to probe the outgoing muon phase space with full acceptance in both angle and momentum.

The double-differential $\nu_{\mu} \mathrm{CC}$ cross section is reported as a function of reconstructed kinematic variables, and obtained using a "forward folding" approach $[23,25]{ }^{1}$ Figure 3 shows the measurement compared to a number of neutrino interaction generator predictions. The

1 A "forward-folding" approach involves publishing data without correcting for detector resolution effects, but pub- 


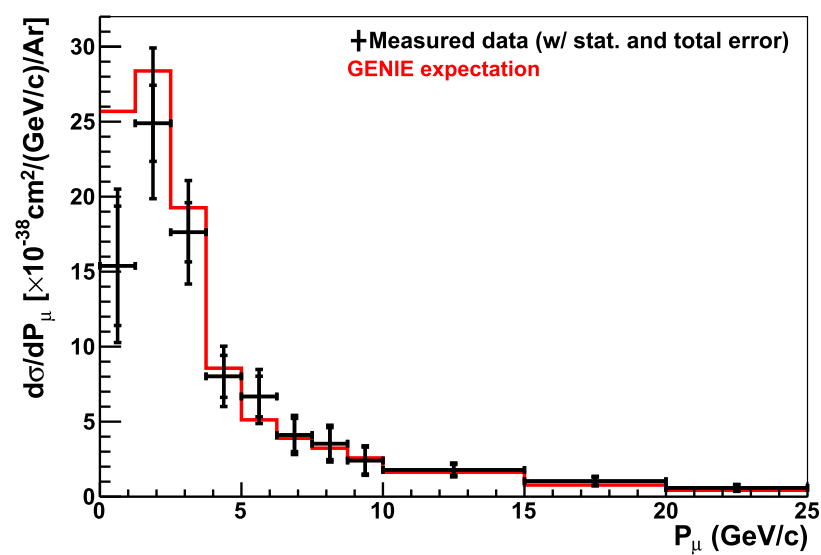

Fig. 1 Flux-integrated differential CC inclusive cross section measured in the ArgoNeuT detector, with NuMI in neutrino mode, as a function of reconstructed muon momentum

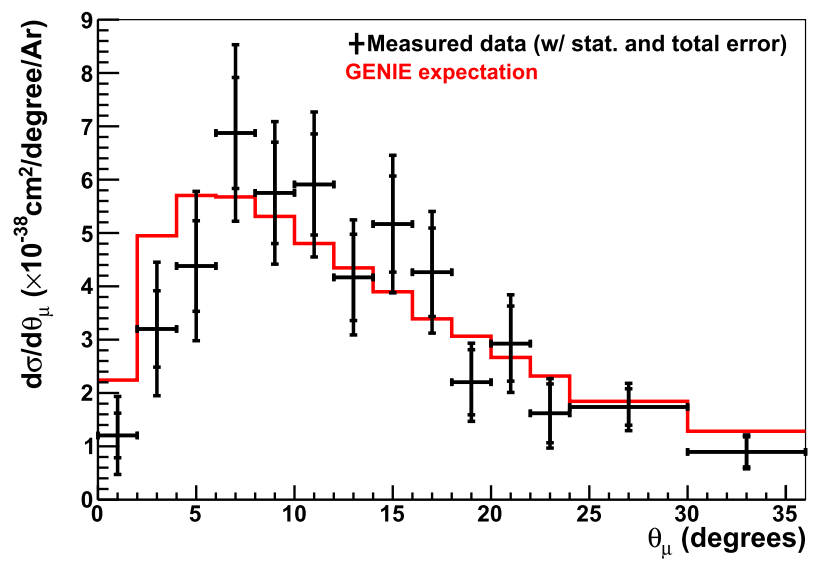

(Left) and scattering angle (Right). Data are compared with a prediction from GENIE v2.6. Figures from [21]
Fig. 2 Flux-integrated differential CC inclusive cross sections measured in the ArgoNeuT detector, with NuMI in antineutrino mode, as a function of reconstructed $\mu^{-}$ (Left) $/ \mu^{+}$(Right) momentum (Bottom) and scattering angle (Top). Figures from [22]
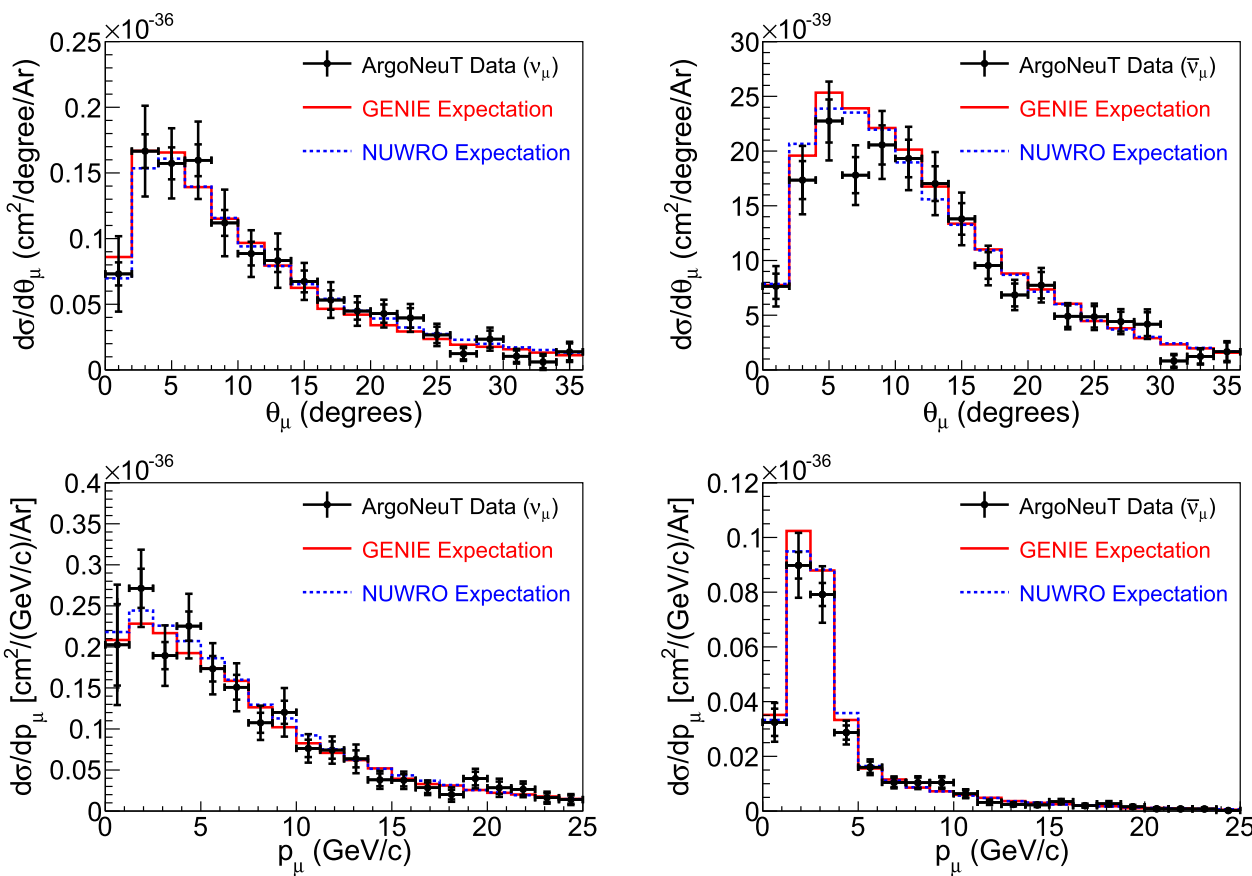

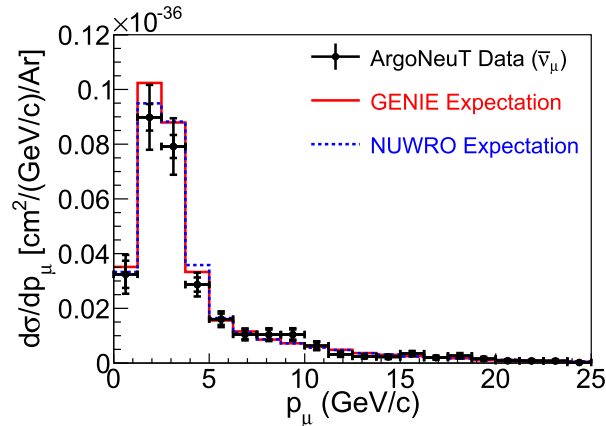

largest disagreements between data and the predictions are in the high-momentum bins in the two most forward-going muon angular bins $\left(0.86 \leq \cos \theta_{\mu} \leq 94\right.$ and $\left.0.94 \leq \cos \theta_{\mu} \leq 1\right)$. In this region the estimated backgrounds are low, and the uncertainty on the measurement is small. $\chi^{2}$ values are calculated between data and the predictions, taking into account the full covariance matrix with off-diagonal elements; the lowest $\chi^{2}$ is found for the GENIE v3 prediction, $\chi^{2}=$ $103.9 / 42$ bins. GENIE v3 predicts a lower cross section in the forward direction than GENIE v2.12.10 (the model used for background simulation and efficiency estimation in this analysis); this is driven predominantly by adopting the local Fermi gas nuclear initial state model, and (to a lesser extent) the inclusion of the random phase approximation (RPA) correction. These effects have the largest impact for low neutrino energies and heavy nuclear targets, so it is expected that these data may be more sensitive to these effects than measurements using lighter target nuclei. Further investigation of these effects will be pursued in two ways: through measurements of exclusive final states, aiming to detangle the impacts of different interaction processes and nuclear effects; and through improved measurement of the inclusive cross section with reduced systematic uncertainties (in particular, the uncertainties on detector modeling for all MicroBooNE analyses presented in this article are large, and have been the focus of significant development efforts by the collaboration $[26-29])$.

lishing matrices that can be used to apply detector resolution effects to predictions. 

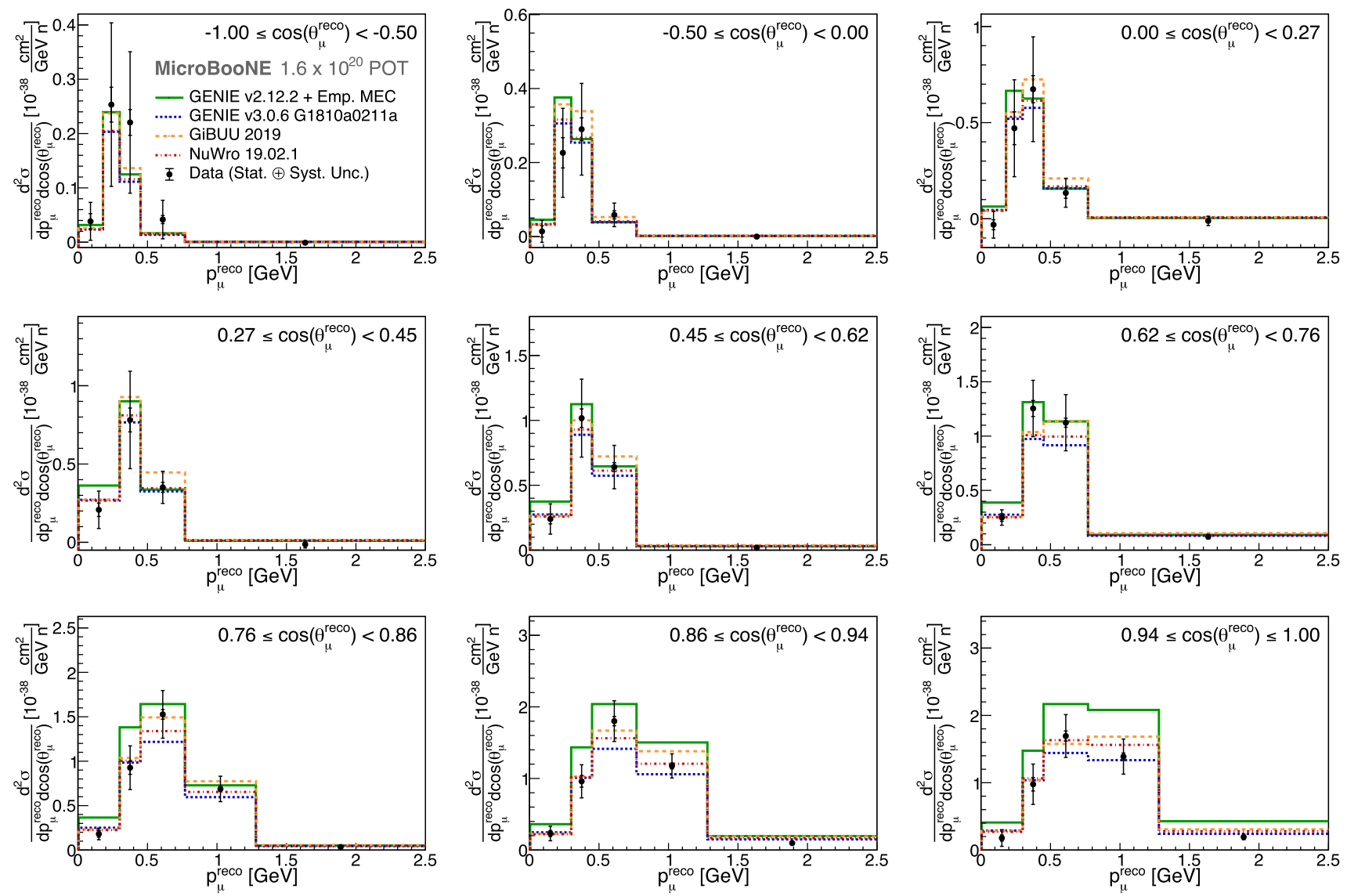

Fig. 3 Flux-integrated double-differential CC inclusive cross section measured in the MicroBooNE detector as a function of reconstructed muon momentum and scattering angle. Figure from [23]

\section{Discussion}

While the MicroBooNE and ArgoNeuT measurements of the inclusive $\mathrm{CC} \nu_{\mu}$ cross section on argon are performed at different neutrino energies-leading to a different underlying composition of the interaction channels - some similarities in the results arise. Overall, both experiments find good agreement with the generators. Interestingly, some tension is present in the forward-angle region of the MicroBooNE measurement (Fig. 3). However, while indications of a similar disagreement are seen in the earlier ArgoNeuT measurement (Fig. 1, left side), the same tendency is not seen in the subsequent measurement (Fig. 2). It should also be noted that the measurements are compared to different generators (in particular, the GENIE versions used in the ArgoNeuT measurement do not include 2p2h models), so it is difficult to compare data-simulation agreement across these data sets.

\section{Focus on protons: measurements of $\nu_{\mu}$ charged-current $0 \pi$ scattering}

Charged-current neutrino interactions that do not produce pions in the final state (commonly called $\mathrm{CC} 0 \pi$ interactions) are of particular interest to many neutrino oscillation experiments $[4,5,30,31]$ because of their relatively simple topology and because they are the dominant interaction type at $<1 \mathrm{GeV}$ neutrino energies. The $\mathrm{CC} 0 \pi$ interaction topology is expected to be composed largely of CCQE and $\mathrm{CC} 2 \mathrm{p} 2 \mathrm{~h}$ interactions, as well as CCRES interactions that produce a pion which is subsequently reabsorbed in the nucleus through finalstate interactions. Both ArgoNeut and MicroBooNE have published measurements of $\mathrm{CC} 0 \pi$ cross section, with particular focus on measuring the protons produced in such interactions, making use of the LArTPC technology's low thresholds for reconstructing and identifying protons.

\subsection{ArgoNeuT measurements of $\mathrm{CC} 0 \pi$ scattering}

\subsection{1 $\nu_{\mu}$ and $\bar{\nu}_{\mu}$ CCO $\pi$ cross section}

Results from the analysis of $\mathrm{CC} 0 \pi$ muon neutrino and antineutrino events in argon collected by the ArgoNeuT experiment on the NuMI beam at Fermilab are presented in Ref. [32]. Measurements of $\nu_{\mu}$ and $\bar{\nu}_{\mu} \mathrm{CC} 0 \pi$ cross sections are reported for different proton multiplicities, using an exposure of $1.25 \times 10^{20} \mathrm{POT}$ in the low-energy antineutrino-mode NuMI beam configuration. An analysis method based on the reconstruction 


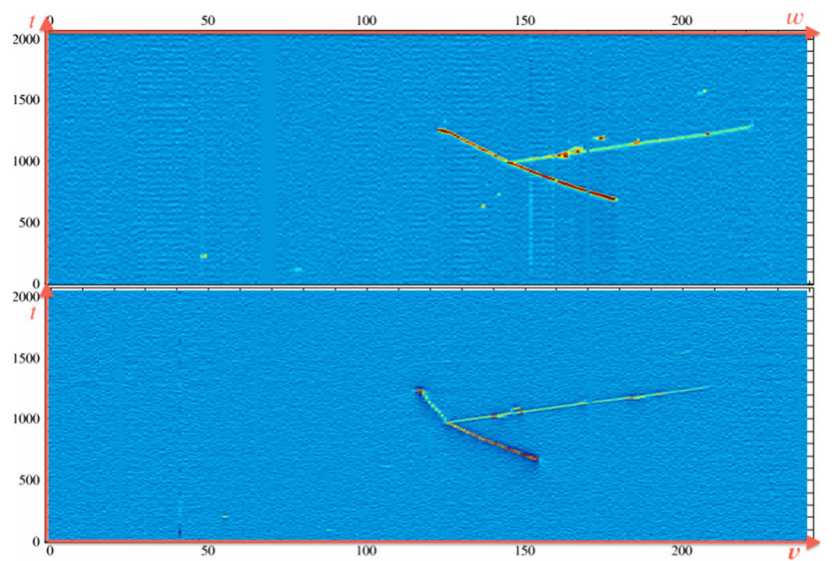

Fig. 4 Two-dimensional views of one of the ArgoNeuT "hammer events", with a forward going muon and a backto-back proton pair. Figure from [33]

of exclusive topologies, fully exploiting the LArTPC detector's capabilities, is used to analyze the events and study nuclear effects in neutrino interactions on argon nuclei. $\mathrm{CC} 0 \pi$ events are characterized by the presence at the vertex of a leading $\mu^{-}$or $\mu^{+}$track, which may be accompanied by one or more highly ionizing tracks (although a proton candidate is not required), and the absence of pion tracks. With a proton reconstruction threshold in ArgoNeuT of $21 \mathrm{MeV}$ kinetic energy, multiple protons accompanying the leading muon and the presence of vertex activity are clearly visible and measured.

\subsubsection{Two-proton $\mathrm{CCO} \pi$ interactions}

Reference [33] reports a measurement of chargedcurrent interactions in the ArgoNeuT detector which produced no pions and exactly two protons in the final state, using the full ArgoNeuT data set $\left(8.5 \times 10^{18}\right.$ POT in the low-energy neutrino-mode NuMI beam configuration, and $1.25 \times 10^{20} \mathrm{POT}$ in the low-energy antineutrino-mode configuration). The kinematics of both protons in these events were studied, showing interesting characteristics. A fraction of the sample detected with ArgoNeuT is found with the two protons in a strictly back-to-back high-momenta configuration in the final state ("hammer events", see Fig. 4). Another equivalent fraction is found to be compatible with a reconstructed back-to-back configuration of a np pair in the initial state inside the nucleus. The detection of these two subsamples suggests that mechanisms directly involving nucleon-nucleon short range correlated pairs in the nucleus are active and can be efficiently explored in neutrino-argon interactions with LArTPC technology. However, the event statistics from ArgoNeuT are very limited and cannot provide definitive conclusions.

\subsection{MicroBooNE measurements of $\mathrm{CC} 0 \pi$ scattering}

MicroBooNE has reported two measurements of $\mathrm{CC} 0 \pi$ interactions in the BNB neutrino flux: a measurement of interactions that produce one muon, one or more protons, and no pions in the final state [25], and a more exclusive measurement of CCQE-like scattering [34]. Both measurements focus on similar topologies (including muons and protons, and excluding pions), but have different signal definitions and selections, leading to different expected proportions of CCQE, CC2p2h and CCRES interactions in the selected events, and complementary constraints on the interaction models. Both measurements are made in detector-measurable quantities such as individual particles' momenta or directions in order to minimise model dependence. Both measurements have a proton detection threshold of $300 \mathrm{MeV} / \mathrm{c}$ (47 MeV kinetic energy), which is driven by a combination of reconstruction algorithm failures at low momenta, and particle identification (PID) failures for short tracks. In both measurements, the PID uses exclusively the (vertical) collection plane, limiting its efficiency at higher proton angles; a limitation that will be eliminated in future measurements by using all three wire planes for PID.

\subsubsection{Charged-current pionless interactions with protons}

Reference [25] reports a measurement of charged current scattering with protons and no pions in the final state (referred to as "CC0 $\pi \mathrm{Np}$ ". The signal definition includes all events that produce protons within the detector's acceptance and don't produce pions. Though primarily composed of quasi-elastic interactions, this signal definition is sensitive to the presence of $2 \mathrm{p} 2 \mathrm{~h}$ interactions and resonant events with absorbed pions, as well as any other potential interactions that produce protons and not pions. This measurement used a data sample corresponding to $1.6 \times 10^{20}$ POT.

The event selection begins with an inclusive muon neutrino charged-current selection from the analysis described in Sect. 3, which removes most backgrounds and identifies a muon candidate. All non-muon clusters are then required to be proton-like (using a particle identification score PID $_{\text {prot }}$ defined by comparing the reconstructed $\mathrm{d} E / \mathrm{d} x$ to the expected energy deposition of a stopping proton [25]). Secondary proton candidates with fewer than five hits on the collection plane are assumed to be protons, as this particle identification method becomes inaccurate with fewer than five hits.

Although the signal definition allows for any number of protons, the measurement focuses on the most energetic (leading) proton. The measurement follows the same forward-folding strategy as [23], whereby predictions must be smeared to compare to the data, and measurements are made of five kinematic variables: the momentum and scattering angle of both the muon and leading proton, as well as the opening angle between those two particles. 


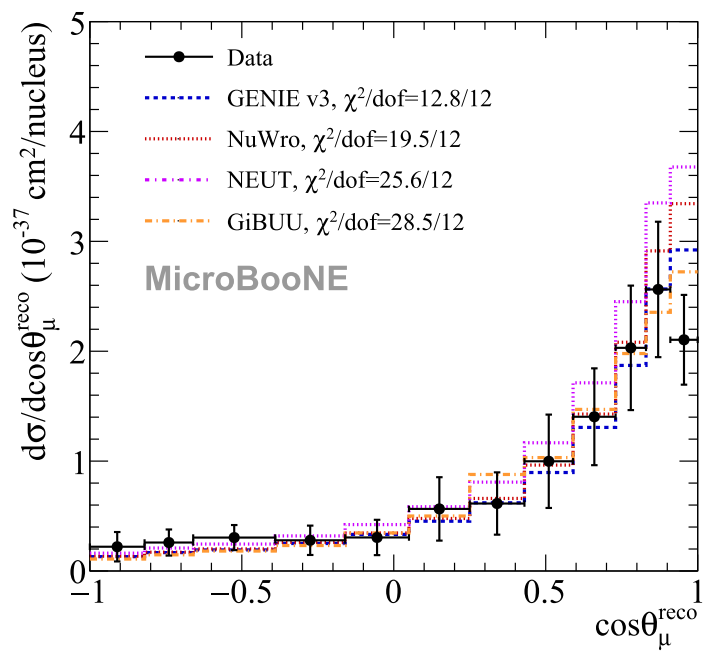

Fig. 5 Flux-integrated single-differential $\mathrm{CC} 0 \pi \mathrm{Np}$ cross section as a function of muon angle. The different lines show predictions from a variety of generators. Figure from [25]

Figure 5 shows the measurement of the muon direction compared to a number of generator predictions. Echoing the observations of the CC inclusive measurement in Sect. 3.2, the data in this more exclusive $\mathrm{CC} 0 \pi$ signal definition also show a suppression at the most forward muon directions. Figure 6 shows comparisons of the same generators across the other four variables. With the exception of the muon momentum distribution, the other variables show reasonable agreement with all models, although tensions can also be seen at the lowest proton momentum.

\subsubsection{Charged-current quasielastic-like scattering}

Reference [34] presents a more exclusive measurement of CCQE-like charged-current muon neutrino scattering on argon with a muon and a single proton in the final state (referred to as "CC1p0 $\pi$ "). The CCQE interaction process can be reasonably well-approximated as a two-body interaction, and these interactions are the focus of many accelerator-based oscillation studies. For this reason, precise measurements of CCQE processes would be valuable inputs to oscillation measurements. However, FSIs make it impossible to measure CCQE processes directly: the proton produced in a CCQE interaction could be reabsorbed in the nucleus, or additional particles (nucleons or pions) could be produced as a result of proton reinteractions. Other processes can also imitate the CCQE signal of one muon and one proton due to FSIs, for example if an additional nucleon or pion is absorbed in the nucleus before exiting. For this reason, Ref. [34] defines 'CCQE-like' events as the signal for this measurement, in which there is one outgoing lepton and exactly one outgoing proton with momentum greater than the reconstruction threshold of $300 \mathrm{MeV} / \mathrm{c}$ (kinetic energy of $47 \mathrm{MeV}$ ). Additional cuts are placed on the signal phase space to further enhance the contribution of CCQE interactions
$\left(\left|\Delta \phi_{\mu p}-180^{\circ}\right|<35^{\circ}\right.$, and $\left|p_{T}^{\mu}+p_{T}^{p}\right|<350 \mathrm{MeV} / \mathrm{c}$, where $p_{T}$ is the reconstructed momentum transverse to the beam) and to keep only regions of phase space where the selection efficiency is high and well-understood $\left(0.3<p_{p}<1.0 \mathrm{GeV} / \mathrm{C}, \cos \theta_{p}>0.15,0.1<p_{\mu}<\right.$ $\left.1.5 \mathrm{GeV} / \mathrm{c},-0.65<\cos \theta_{\mu}<0.95,\left|\Delta \theta_{\mu p}-90^{\circ}\right|<55^{\circ}\right)$.

This measurement was performed using the on-axis BNB neutrino flux, with an exposure of $4.59 \times 10^{19}$ POT. CCQE-like candidate events are selected by identifying pairs of tracks, of which one is classified as a muon candidate and the other as a proton candidate

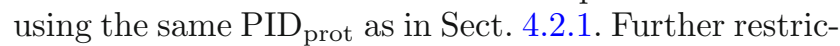
tions are placed on the reconstructed kinematics of the muon and proton, to match the signal phase space.

Figure 7 shows the flux-integrated single-differential CCQE-like cross section as a function of the cosine of the measured muon scattering angle. In general the measurement finds good agreement between the data and predictions from most of the generators, except in the most forward-going bin $\left(0.8<\cos \theta_{\mu}<0.95\right)$; the overprediction at forward-going muon angles shown in Sects. 3.2 and 4.2.1 is also seen in this more exclusive measurement. The fact that the overprediction grows with increasing exclusivity is indicative that this effect is due to nuclear effects impacting quasielasticlike interactions, either in true quasielastic interactions, or in other interactions that mimic the signature. Figure 8 shows the flux-integrated single-differential cross sections as a function of muon momentum, proton momentum, and cosine of the proton scattering angle. Results are shown both for the full phase-space of $\cos \theta_{\mu}$ and for $\cos \theta_{\mu}<0.8$. The data-prediction agreement is improved across all variables when excluding the most forward-going muon bin.

\section{Discussion}

LArTPC technology allows the reconstruction of neutrino interactions producing protons in the final state with never-before-accessible low energy thresholds, in high statistics datasets. This is a powerful line of research when testing nuclear models in neutrino generators, as the multiplicity and kinematics of protons in the final state can be impacted by several nuclear mechanisms, such as short range nuclear correlations and pion nuclear absorption, which have large theoretical uncertainties. The ArgoNeuT measurements already highlight how proton multiplicity is a thorny territory for neutrino generator predictions.

The MicroBooNE measurements show tension in the lepton forward angle, in which the models overpredict the measured data. A similar tension was also observed in the $\mathrm{CC}$ inclusive measurement presented in Sect. 3.2, and this is seen to be more substantial in the more exclusive measurements (with the largest tension in the most exclusive CCQE-like measurement). The high $\cos \theta_{\mu}$ phase space corresponds to low momentumtransfer events, so this may indicate a mismodeling of signal or background events in that phase space (the more exclusive measurements rely more on simulation 

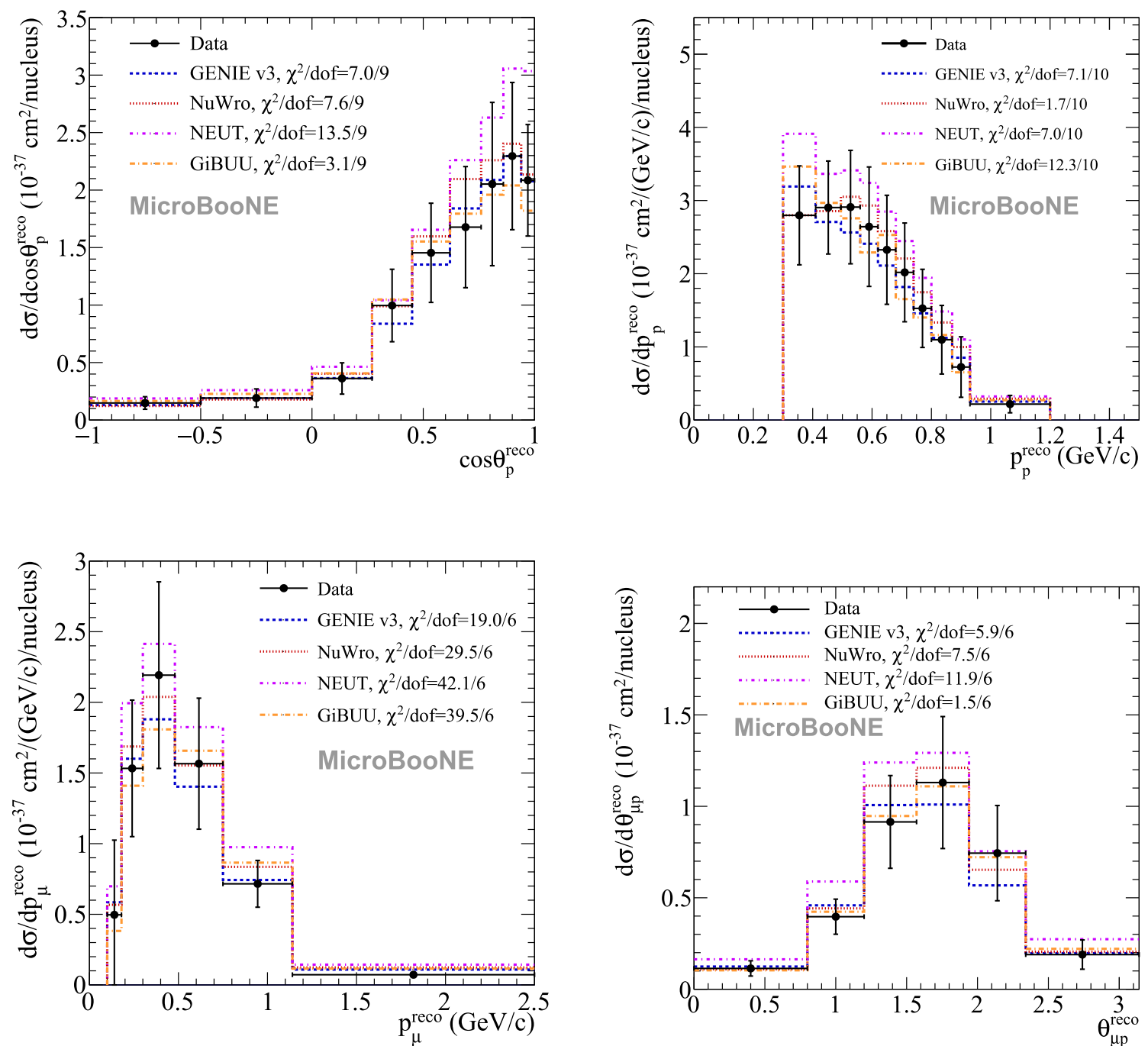

Fig. 6 Flux-integrated single-differential $\mathrm{CC} 0 \pi \mathrm{Np}$ cross section as a function of (top left) proton angle, (top right) proton momentum, (bottom left) muon momentum, and (bottom right) muon-proton opening angle. Figure from [25]

to subtract backgrounds). These results justify further investigation into the behaviour of cross sections at low four-momentum transfer, and future measurements may learn more about this region by reducing the proton detection threshold.

ArgoNeuT's two-proton channel measurement is particularly interesting for the implications of exploring short-range correlations in neutrino scattering, and this will be extensively studied in a future dedicated search with MicroBooNE. Further investigation of $\mathrm{CC} 0 \pi$ topologies will also include analysis of transverse imbalance variables and other derived quantities that are more sensitive to nuclear models.

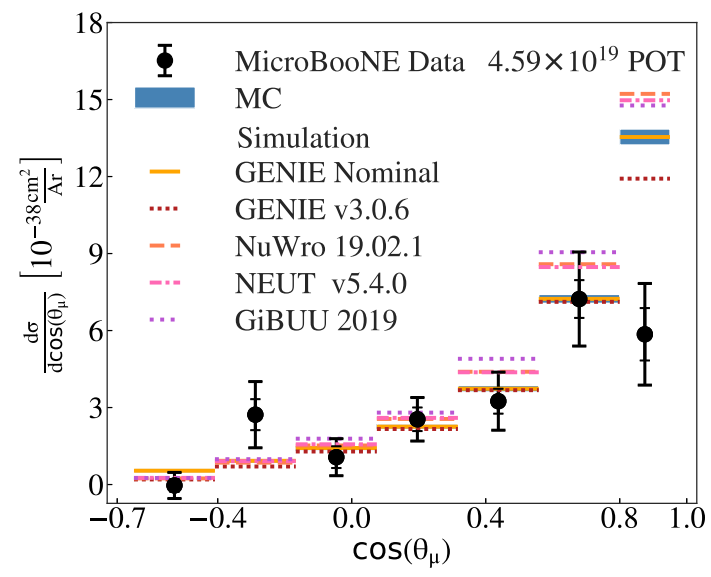

Fig. 7 Flux-integrated single-differential $\mathrm{CC} 1 \mathrm{p} 0 \pi$ cross section as a function of the cosine of the measured muon scattering angle. Colored lines show the predictions of different event generators for comparison (the lines labeled ' $\mathrm{MC}$ ' and 'GENIE Nominal' both show the GENIE v2.12.2 prediction). Figure from [34] 


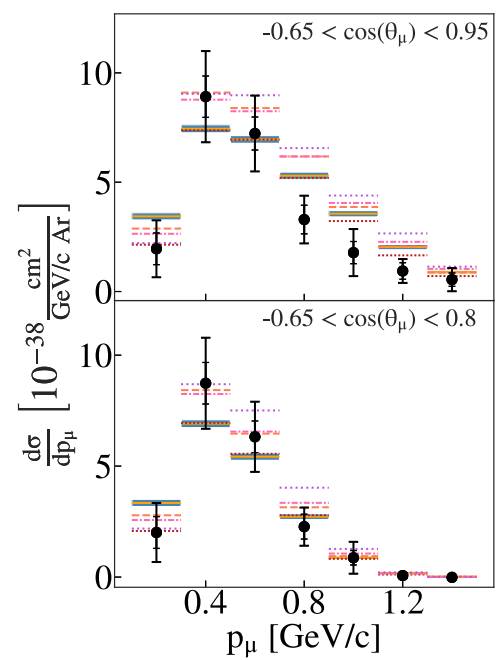

Fig. 8 Flux-integrated single-differential $\mathrm{CC} 1 \mathrm{p} 0 \pi$ cross section as a function of measured muon momentum (left), proton scattering angle (middle), and proton momentum

\section{Focus on pions: neutrino-induced single pion production}

\section{$5.1 \nu_{\mu}$ charged pion production}

Charged-current pion production is an important process in few-GeV neutrino-nucleus interactions, where the underlying interactions mainly proceed through the excitation of nucleon resonances, deep inelastic scattering or coherent scattering of the neutrino on the entire nucleus. A precise measurement of the observed charged pion rates and understanding of the underlying physics processes is crucial for future neutrino oscillation measurements, particularly at DUNE [4] where single pion production is expected to be the dominant interaction process.

\subsubsection{ArgoNeuT measurement of charged-current single pion production}

Reference [35] reports the first $\mathrm{CC} 1 \pi^{ \pm}$differential cross section measurement on argon using the ArgoNeuT detector. The signal is defined to be a charged-current $\nu_{\mu}$ or $\bar{\nu}_{\mu}$ interaction in the detector, with only one charged pion above $100 \mathrm{MeV} / c$ momentum exiting the target nucleus. The measurements are based on data taken with the NuMI beam line operating in the lowenergy antineutrino-mode configuration, corresponding to $1.25 \times 10^{20} \mathrm{POT}$. The event selection requires that there is at least one track in ArgoNeuT that is matched to a track in the MINOS detector. The matched track is identified as the muon track. A boosted decision tree (BDT) is trained using the calorimetric and topological information in the TPC to select events with a single charged pion in the final state. The selection efficiency is approximately $30 \%$ for both the $\nu_{\mu}$ and $\bar{\nu}_{\mu} \mathrm{CC} 1 \pi^{ \pm}$ samples. The purity is $36 \%$ (56\%) for the selected $\nu_{\mu}$ $\left(\bar{\nu}_{\mu}\right) \mathrm{CC} 1 \pi^{ \pm}$sample. Figure 9 shows the ArgoNeuT $\nu_{\mu}$ and $\bar{\nu}_{\mu} \mathrm{CC} 1 \pi^{ \pm}$differential cross sections compared to four neutrino generators: GENIE, NuWro, GiBUU and NEUT. The GiBUU predictions are in a reasonable agreement with the measured cross sections. The NuWro, NEUT and GENIE predictions are higher than the measured cross section reported in the paper.

\subsubsection{ArgoNeuT measurement of coherent charged pion production}

Neutrinos can produce single pion final states by coherently scattering from the entire nucleus. Both neutral current and charged current coherent-scattering processes are possible. In these interactions, the squared four-momentum transfer to the target nucleus, $|t|$, is small so the nucleus remains unchanged. Reference [36] reports the $\mathrm{CC}$ coherent pion production from muon neutrinos and antineutrinos on argon using the ArgoNeuT detector:

$$
\begin{aligned}
& \nu_{\mu}+\mathrm{Ar} \rightarrow \mu^{-}+\pi^{+}+\mathrm{Ar} \\
& \bar{\nu}_{\mu}+\mathrm{Ar} \rightarrow \mu^{+}+\pi^{-}+\mathrm{Ar}
\end{aligned}
$$

where the low $|t|$ condition entails that the pions and muons are forward going with respect to the incoming neutrino direction. The measurements are based on data taken with the NuMI beam line operating in the low-energy antineutrino-mode configuration, corresponding to $1.25 \times 10^{20} \mathrm{POT}$. The event selection requires exactly two tracks consistent with being minimum-ionizing particles (MIPs), with one track matched to a track in the MINOS detector as the muon candidate. The lack of any particles other than the muon and the pion emerging from the vertex is further reinforced by rejecting events with high charge 

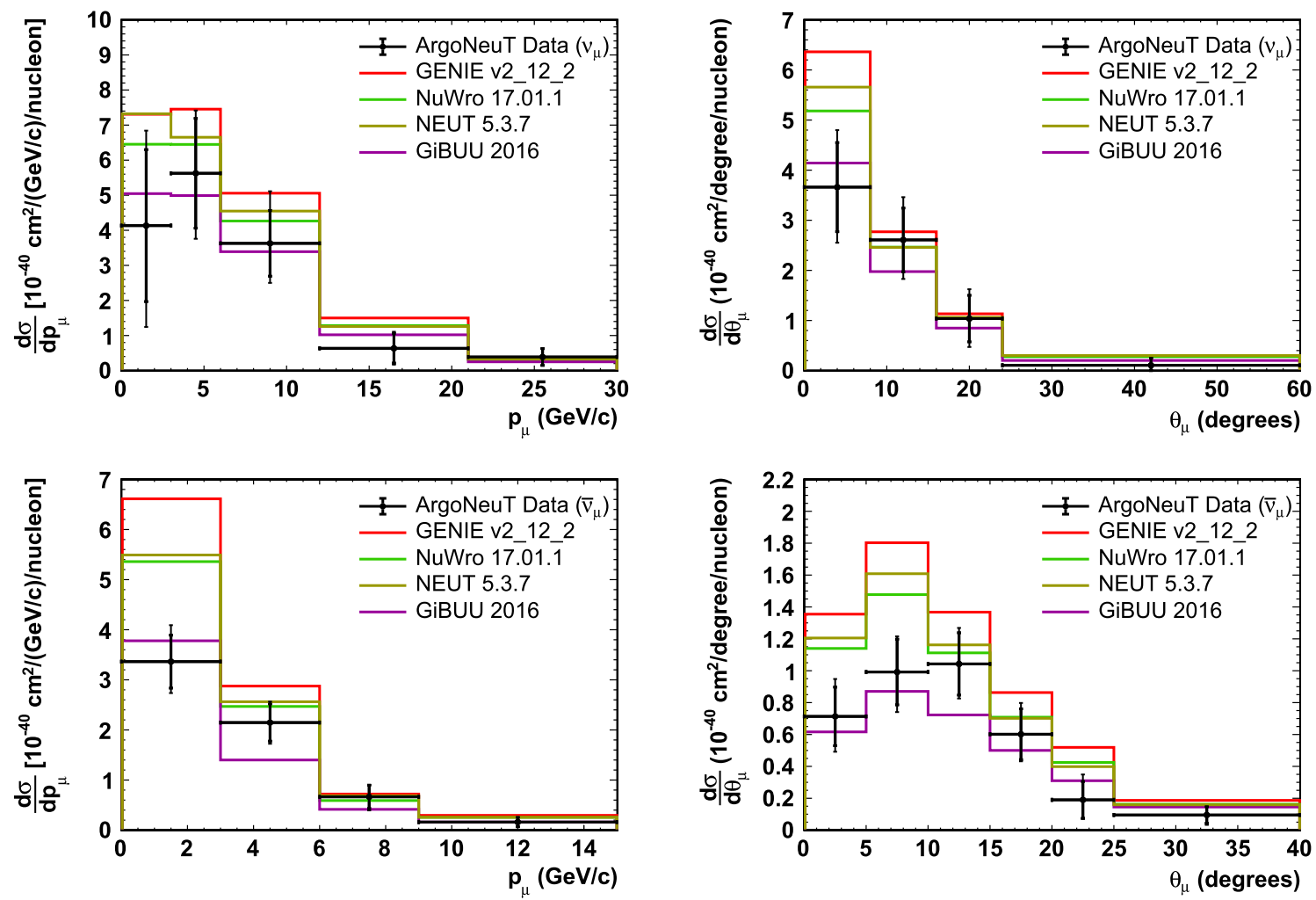

Fig. 9 ArgoNeuT $\nu_{\mu}$ and $\bar{\nu}_{\mu}$ CC1 $\pi^{ \pm}$differential cross sections compared to GENIE, NuWro, GiBUU and NEUT. Thick error bars refers to statistical errors while thin error bars refers to statistical and systematic errors summed together. Figure from $[35]$

deposition around the vertex. A BDT is trained using the calorimetric and kinematic information to select events with low $|t|$. The selection efficiency is approximately $20 \%$ for both the $\nu_{\mu}$ and $\bar{\nu}_{\mu}$ coherent charged pion samples. The purity is close to $100 \%$. A comparison between the ArgoNeuT's CC coherent pion cross section measurements, existing data, and the predictions from GENIE and NuWro is shown in Fig. 10. The antineutrino measurement agrees well with the ReinSeghal model [37] while the measured neutrino cross section deviates by $\sim 1.2 \sigma$.

\section{$5.2 \nu_{\mu}$ neutral pion production}

Neutral pions produced in neutrino interactions generally decay to two photons inside the detector. Photons, like electrons, produce electromagnetic showers in LArTPC detectors; while photon-induced showers can be distinguished from electron-induced showers using characteristics such as distance between the neutrino interaction vertex and the shower start and the energy deposited per unit length, $\mathrm{d} E / \mathrm{d} x$ (as discussed in Sect. 6), photons from $\pi^{0}$ decay may still form important backgrounds to $\nu_{e}$ appearance oscillation searches due to reconstruction failures. Therefore, it is important that these interactions be understood. Because of the well-known mass of the $\pi^{0}$, neutral pions can also form a "standard candle" for tests of the shower energy resolution in LArTPC reconstruction.

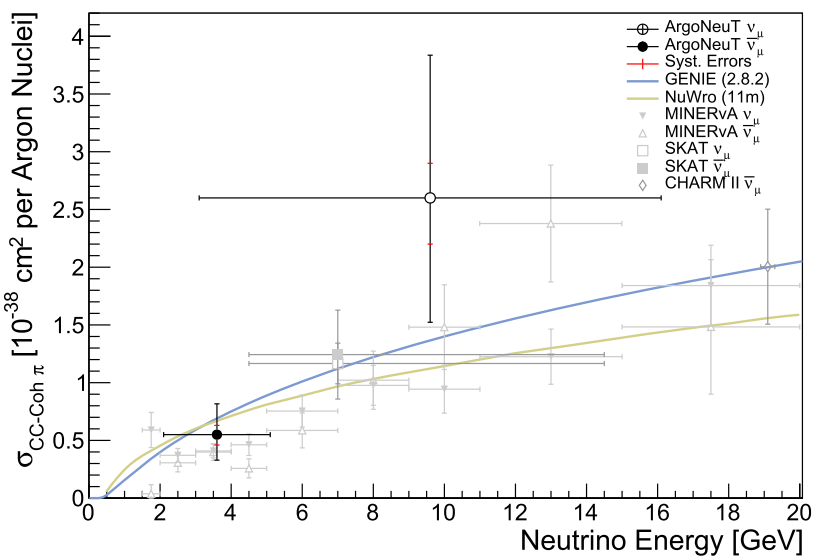

Fig. 10 ArgoNeuT's CC coherent pion cross section measurements compared to model predictions and measurements from the SKAT [38], CHARM [39] and MINERvA [40] experiments. These measurements are scaled to Argon assuming the $A^{1 / 3}$ dependence from the Rein-Seghal model. Figure from [36]

\subsubsection{ArgoNeuT measurement of neutral-current neutral pion production}

Reference [41] reports ArgoNeuT's measurement of the cross-section for neutral current (NC) $\pi^{0}$ production in $\nu_{\mu}$-argon interactions, using an exposure of $1.25 \times 10^{20}$ POT in the low-energy antineutrino-mode NuMI beam 

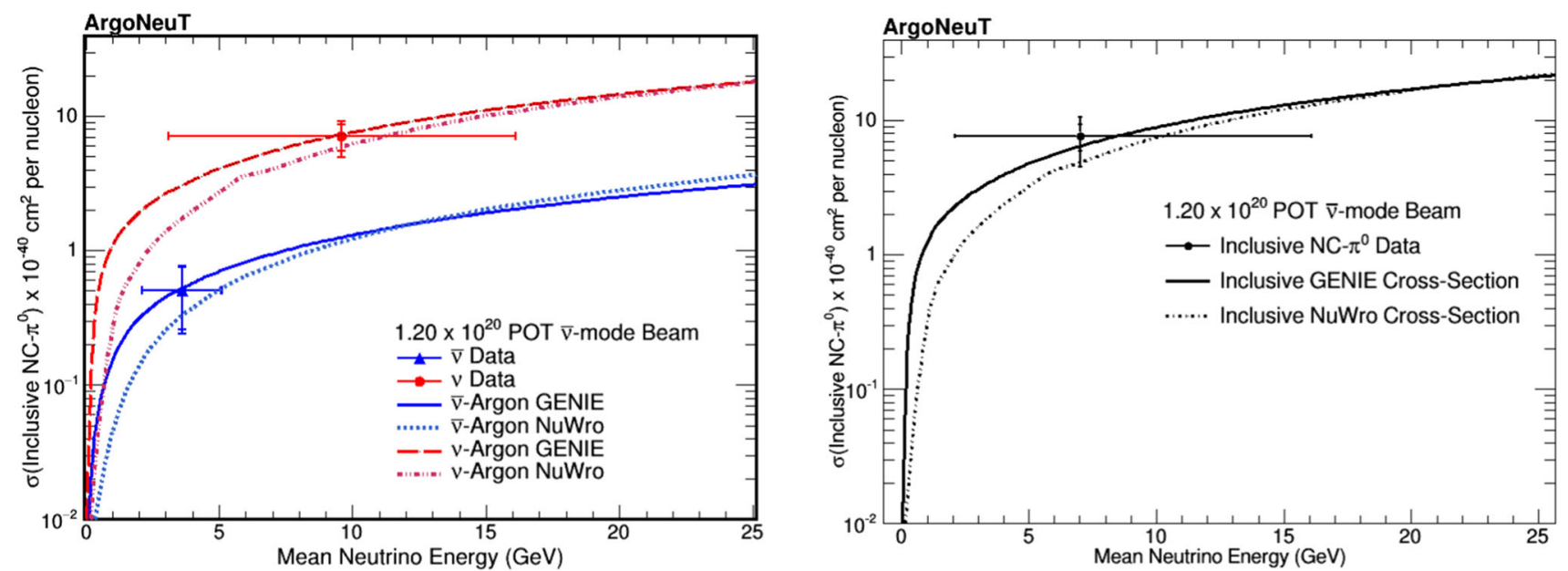

Fig. 11 The NC $\pi^{0}$ cross-section measured in ArgoNeuT compared with predictions from GENIE (solid) and NuWro (dashed), Left: for neutrino (red) and antineutrinos (blue) separately, and Right: for a combined $\nu_{\mu}+\bar{\nu}_{\mu}$ measurement. Figures from [41]

configuration. The event selection requires the presence of two reconstructed electromagnetic shower candidates consistent with a $\pi^{0} \rightarrow \gamma \gamma$ decay. Consistency with the $\gamma$ hypothesis is obtained by requiring that at least $75 \%$ of the first $4 \mathrm{~cm}$ of each shower candidate presents a $\mathrm{d} E / \mathrm{d} x$ value greater than $3.5 \mathrm{MeV} / \mathrm{cm}$. The selection further requires the absence of additional reconstructed tracks to reduce the contribution of charged-current interactions. Candidate interactions are required to originate within a fiducial volume that is the same as that defined for the $\mathrm{CC}$ inclusive analyses described in Sect. 3.1. Given the very small size of the ArgoNeuT detector almost none of the candidate showers are fully contained; thus, the analysis develops a series of model-driven corrections leveraging the angular and deposited energy information reconstructed in the contained part of the shower to estimate the true energy of each shower. The analysis is further complicated by the impossibility to distinguish between neutrino- and antineutrino-induced NC $\pi^{0}$ production-both sizeable contributions in the antineutrino-mode NuMI beam. As such, the final result-shown in Fig. 11-reports both a combined $\nu_{\mu}+\bar{\nu}_{\mu}$ cross-section (right) as well as separate neutrino and antineutrino cross-sections that assumed a fractional composition in the data that matched that of the GENIE (v2.8.0) model.

\subsubsection{MicroBooNE measurement of charged-current neutral pion production}

While neutral-current $\pi^{0}$ production forms the majority of backgrounds to $\nu_{\mu} \rightarrow \nu_{e}$ oscillation searches, the spectrum and rate of $\pi^{0}$ production can be sculpted by FSI in the nucleus, so a good understanding of the impact of this is needed to estimate this background. To first order, assuming isospin symmetry, the interaction of a neutral pion with the residual nucleus is the same for both the neutral-current interaction mode an the charged-current interaction mode, so charged-current interactions that produce a $\pi^{0}$ are a useful way of con- straining the impact of FSI on this important background. Reference [42] reports the first measurement of $\nu_{\mu}$ CC single $\pi^{0}$ production on argon, using the MicroBooNE detector and a BNB exposure of $1.62 \times 10^{20}$ POT. The measurement is performed using an inclusive final-state topology of at least one photon from a $\pi^{0}$ decay exiting the nucleus, in addition to a muon, and any number of other particles.

Events are selected based on the presence of reconstructed showers, which are assumed to be produced by photons, and a reconstructed muon track. Tracklike topologies are rejected, to reduce cosmic ray contamination, although this leads to a reduced showerreconstruction efficiency for photons with kinetic energies below $50 \mathrm{MeV}$ (which can appear more track-like). This has an impact on the selection efficiency, because the energy distribution for sub-leading photons produced by the decays of $\pi^{0} \mathrm{~s}$ at $\mathrm{BNB}$ neutrino energies peaks below $50 \mathrm{MeV}$. When requiring two showers to be reconstructed, the selection has an estimated purity of $64 \%$ and efficiency of $6 \%$; when requiring at least one reconstructed shower, the selection has an estimated purity of $56 \%$ and efficiency of $16 \%$. The result, calculated using the single-photon selection and shown in Fig. 12, is a flux-integrated total cross section for semiinclusive CC single $\pi^{0}$ production on argon. A slight deficit $(1.2 \sigma)$ is seen relative to the GENIE prediction for argon. The measurement is also compared to measurements of exclusive CC single $\pi^{0}$ production in the MiniBooNE [43] and Argonne National Laboratory (ANL) bubble chamber [44] experiments; the reasonable agreement of all three measurements with model predictions indicates that the nuclear scalings implemented in these models are applicable, within uncertainties, for neutrino-argon scattering.

\section{Discussion}

ArgoNeuT and MicroBooNE have thus far performed complementary explorations of neutrino interactions 

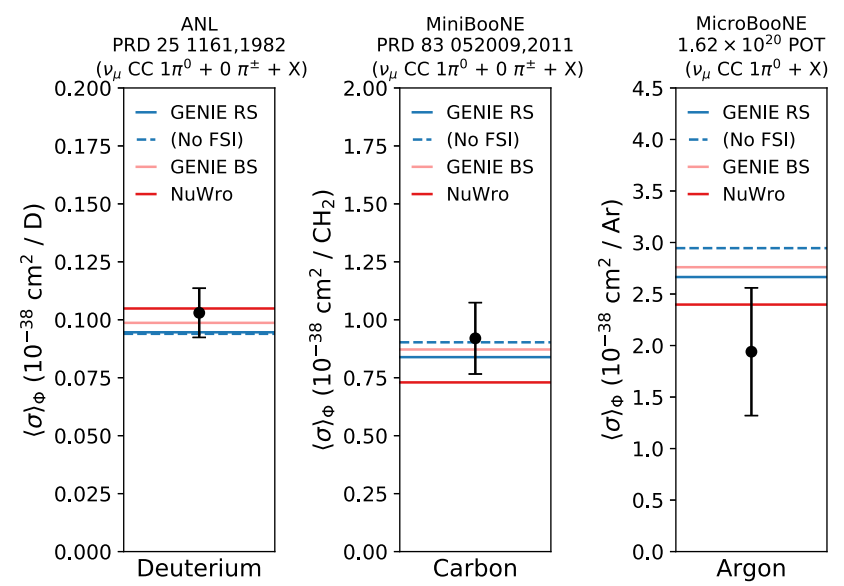

Fig. 12 Total flux integrated $\nu_{\mu}$ CC single $\pi^{0}$ production cross section measured in the ANL bubble chamber [44], MiniBooNE [43], and MicroBooNE [42]. These are compared to the GENIE v2.12.00 prediction with the ReinSehgal model (RS) [45], the RS model with FSI removed (No FSI), an alternative GENIE prediction using the BergerSehgal model (BS) [46], and the NuWro v18.02.01 default prediction. Figure from [42]

producing pions in the final state in terms of signal definition. ArgoNeuT explored the $\mathrm{CC} 1 \pi^{ \pm}$channel, $\mathrm{CC}$ coherent charged-pion production, and the NC production of $\pi^{0}$, while MicroBooNE has focused on the production of $\pi^{0}$ in $\mathrm{CC}$ interactions. The ArgoNeuT result for the $\mathrm{CC} 1 \pi^{ \pm}$channel is particularly interesting as it presents tension with almost all generators in the low momentum and low angle region for both $\nu_{\mu}$ and $\bar{\nu}_{\mu}$. When exploring coherent pion production, ArgoNeuT measurements for the antineutrino data are in good agreement with the Rein-Seghal model for neutrino excitation of baryon resonances but present a slight deviation in the neutrino data. Both coherent and resonant $\mathrm{CC} 1 \pi^{ \pm}$channels will be explored further in MicroBooNE with dedicated high-statistics searches. Hints of tension can also be found in the MicroBooNE's measurement of the total flux integrated $\nu_{\mu}$ CC single $\pi^{0}$ production cross section, where the generators' prediction tends to be higher than the measured value (although within estimated uncertainties).

\section{$6 \nu_{e}$ and $\bar{\nu}_{e}$ scattering}

The measurement of electron neutrinos appearing in a muon-neutrino beam is essential to measure the neutrino mass ordering [47] and to search for CP violation in the neutrino sector $[4,48]$ in long-baseline experiments. In short-baseline experiments, the analogous signature has the potential to reveal the existence of sterile neutrinos [5].

Generally, accelerator neutrino oscillation experiments use $\nu_{\mu}$ interactions to constrain the $\nu_{e}$ flux and cross section models $[18,49]$, mitigating the lack of pre- cise electron-neutrino cross section measurements. Any uncertainty on the $\nu_{e} / \nu_{\mu}$ cross section ratio weakens the reliability of such a procedure. In principle under the assumption of lepton universality, the only difference between $\nu_{e}$ and $\nu_{\mu}$ interactions is in the final state lepton mass. However, that different mass also leads to differences in the cross section through radiative corrections, as well as through changes to form factors. The impact of radiative corrections is expected to be approximately 10\% [50]. Additionally, nuclear effects in general depend on the energy and momentum transferred to the nucleus, which will be different due to the lepton mass, and therefore uncertainties in those nuclear effects can increase the uncertainty on the $\nu_{e} / \nu_{\mu}$ cross section ratio. Recent theoretical calculations predict large differences between the $\nu_{e}$ and $\nu_{\mu}$ cross sections, particularly for forward-going leptons in the sub$\mathrm{GeV}$ range [51]. Independent direct measurements of electron-neutrino cross sections on the target medium of choice for future neutrino oscillation experiments are therefore of paramount importance to further inform our understanding of different flavor neutrino interactions and perform oscillation searches to the desired precision.

\subsection{ArgoNeuT measurement of inclusive CC $\nu_{e}$ and $\bar{\nu}_{e}$ cross section}

Reference [52] reports measurement of the combined $\nu_{e}+\bar{\nu}_{e}$ flux-averaged charged-current inclusive cross section performed using the on-axis flux from the NuMI beam in the low-energy antineutrino-mode configuration, corresponding to $1.25 \times 10^{20}$ POT. In the small ArgoNeuT detector, showers are rarely completely contained; this prohibits the use of total charge for complete shower characterization and energy reconstruction. Instead, charge ratios characterizing the shape and evolution of each candidate electron shower are used to select electrons. A BDT is constructed using the charge ratios as well as the shower angle and vertex $\mathrm{d} E / \mathrm{d} x$. The event selection is expected to have a $10.5 \%$ efficiency with a $78.9 \%$ purity based on studies of simulation. A flux-averaged $\nu_{e}+\bar{\nu}_{e}$ total cross section and a differential cross section as a function of lepton angle are extracted using $13 \nu_{e}$ and $\bar{\nu}_{e}$ events identified with fully-automated selection and reconstruction. A total cross section of (1.04 \pm 0.38 (stat. $)_{-0.23}^{+0.15}$ (syst.) $) \times 10^{-36} \mathrm{~cm}^{2}$ on argon $\left(\left\langle E_{\nu_{e}}\right\rangle=\right.$ $10.5 \mathrm{GeV}$ and $\left.\left\langle E_{\bar{\nu}_{e}}\right\rangle=4.3 \mathrm{GeV}\right)$ was extracted, consistent with the GENIE v2.12.10 expectation. Figure 13 shows the ArgoNeuT $\nu_{e}+\bar{\nu}_{e}$ CC differential cross section for electron/positron angle with respect to the neutrino beam compared to the GENIE prediction. These are the first measurements of electron neutrino scattering cross sections on argon, which provide a good validation of the shower reconstruction technique and the modeling of electron neutrino cross sections using the same target nucleus and over the same energy range that will be used by DUNE. 


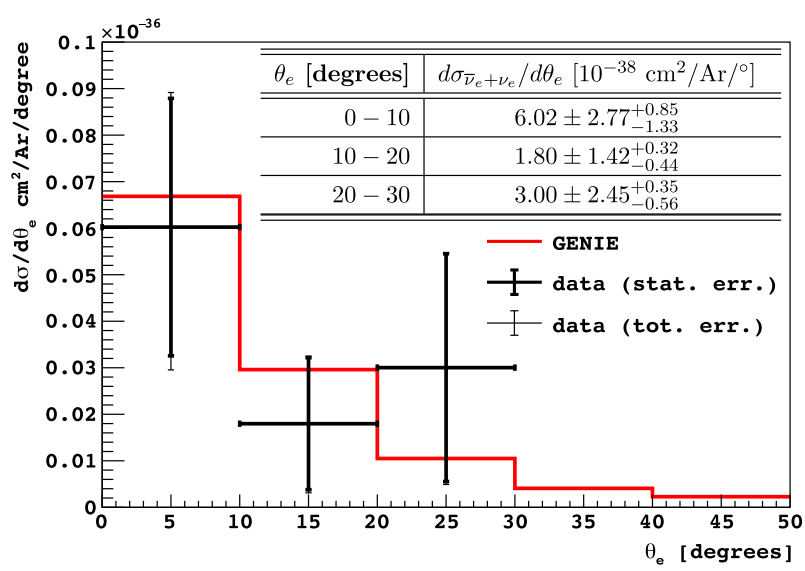

Fig. 13 The ArgoNeuT $\nu_{e}+\bar{\nu}_{e}$ CC differential cross section for electron/positron angle with respect to the neutrino beam compared to the GENIE prediction. Figure from [52]

\subsection{MicroBooNE measurement of inclusive CC $\nu_{e}$ and $\bar{\nu}_{e}$ cross section}

Reference [20] reports measurement of the combined $\nu_{e}+\bar{\nu}_{e}$ flux-averaged charged-current inclusive cross section performed using the off-axis flux from the NuMI beam in the medium energy neutrino mode, as measured in the MicroBooNE detector. The integrated neutrino flux above $250 \mathrm{MeV}$ has an average of $0.905 \mathrm{GeV}$. The measurement selects 214 candidate $\nu_{e}+\bar{\nu}_{e}$ interactions with an estimated exposure of $2.4 \times 10^{20}$ protons on target. Given the estimated purity of $38.6 \%$, this implies the observation of $80 \nu_{e}+\bar{\nu}_{e}$ interactions in argon.

The analysis follows a staged approach for background removal. The initial stages of the selection focus on reducing cosmic ray contamination, by a combined strategy that leverages the timing of the interaction, correlations between optical and TPC information, and quality requirements on the reconstructed interaction vertex. The identification of a reconstructed electromagnetic shower abates backgrounds from $\nu_{\mu} \mathrm{CC}$ interactions. The characteristics of the leading shower such as $\mathrm{d} E / \mathrm{d} x$, Molière angle, and distance from the reconstructed neutrino interaction vertex are leveraged to select $\nu_{e} \mathrm{CC}$ inclusive interactions. Indeed, the analysis includes a demonstration of a fully-automated $\mathrm{d} E / \mathrm{d} x$ based particle discrimination technique for electronand photon-induced showers in a LArTPC neutrino detector, as well as a study of electron and photon separation. The example $\mathrm{d} E / \mathrm{d} x$ distribution for electromagnetic showers reported by the analysis can be found in Fig. 14.

While the selection accomplishes an overall decrease in the cosmic ray contamination of a factor of $10^{5} \mathrm{com}-$ pared to the initial sample, the main background for this first $\nu_{e}$ analysis in MicroBooNE remains cosmic ray contamination ( $42 \%$ of all selected events). Significantly higher purity is expected in underground detectors, as well as with next-generation reconstruction algorithms where cosmic rays are rejected via improved light and charge matching, topology reconstruction and CRT cosmic ray tagging. The leading uncertainties for the measurements are the detector modeling (23\%), the modeling of hadron production for the flux prediction (22\%) and statistical uncertainty (22\%); all are expected to reduce with future iterations.

The charged-current $\nu_{e}+\bar{\nu}_{e}$ flux-averaged total cross section is measured to be $6.84 \pm 1.51$ (stat.) \pm 2.33 (sys.) $\times 10^{-39} \mathrm{~cm}^{2} /$ nucleon. Figure 14 shows the measurement compared to a number of predictions, in particular to GENIE v2.12.10 (the model used for background simulation and efficiency estimation in this analysis), GENIE v3.0.6 and NuWro v19.02.1, showing good agreement with all.

\section{Discussion}

MicroBooNE and ArgoNeuT both measured the inclusive CC $\nu_{e}+\bar{\nu}_{e}$ cross section on argon analyzing neutrinos from the NuMI beam. Despite using the same beam, there are significant differences in the measured neutrino flux. The average flux energy is much higher for the ArgoNeuT measurement given its position onaxis to the NuMI beam. As a consequence, the dominant interaction channels predicted in for the inclusive channel are DIS and RES for ArgoNeuT, compared to predominantly CCQE for MicroBooNE. The neutrino to antineutrino flux ratio is also different given the configurations of the NuMI beam for the data employed: antineutrino mode for ArgoNeuT and neutrino mode for MicroBooNE. Both experiments find similar agreement to the theory predictions for the flux-averaged cross section: the agreement between the GENIE v2.12.2 used in MicroBooNE and the GENIE v2.12.10c used in ArgoNeuT is equivalent. Following the first ArgoNeuT measurement of differential electron neutrino cross section in lepton angle, a more detailed exploration of inclusive and exclusive electron neutrino interaction channels is expected from MicroBooNE's higher statistics data sample.

\section{Opportunities in neutrino-argon interaction measurements}

\subsection{New analysis techniques}

Future LArTPC measurements will continue to develop analysis techniques that take advantage of the detectors' low thresholds and high-resolution reconstruction. Future measurements from MicroBooNE will benefit from improved cross-section, detector, and cosmic ray modeling, and associated reductions in systematic uncertainties (particularly those associated with the detector model). This will allow more precise measurements of neutrino interactions, including detailed measurements of hadronic final states and variables not 
Fig. 14 (Left)

Flux-averaged $\nu_{e}+\bar{\nu}_{e}$ charged-current inclusive cross section on argon performed using the off-axis flux from the NuMI beam at MicroBooNE. (Right) Distribution of $\mathrm{d} E / \mathrm{d} x$ of leading showers for neutrino candidates. Electrons are gathered in the MIP peak of $2 \mathrm{MeV} / \mathrm{cm}$, while most photons around $4 \mathrm{MeV} / \mathrm{cm}$. Figures from $[20]$
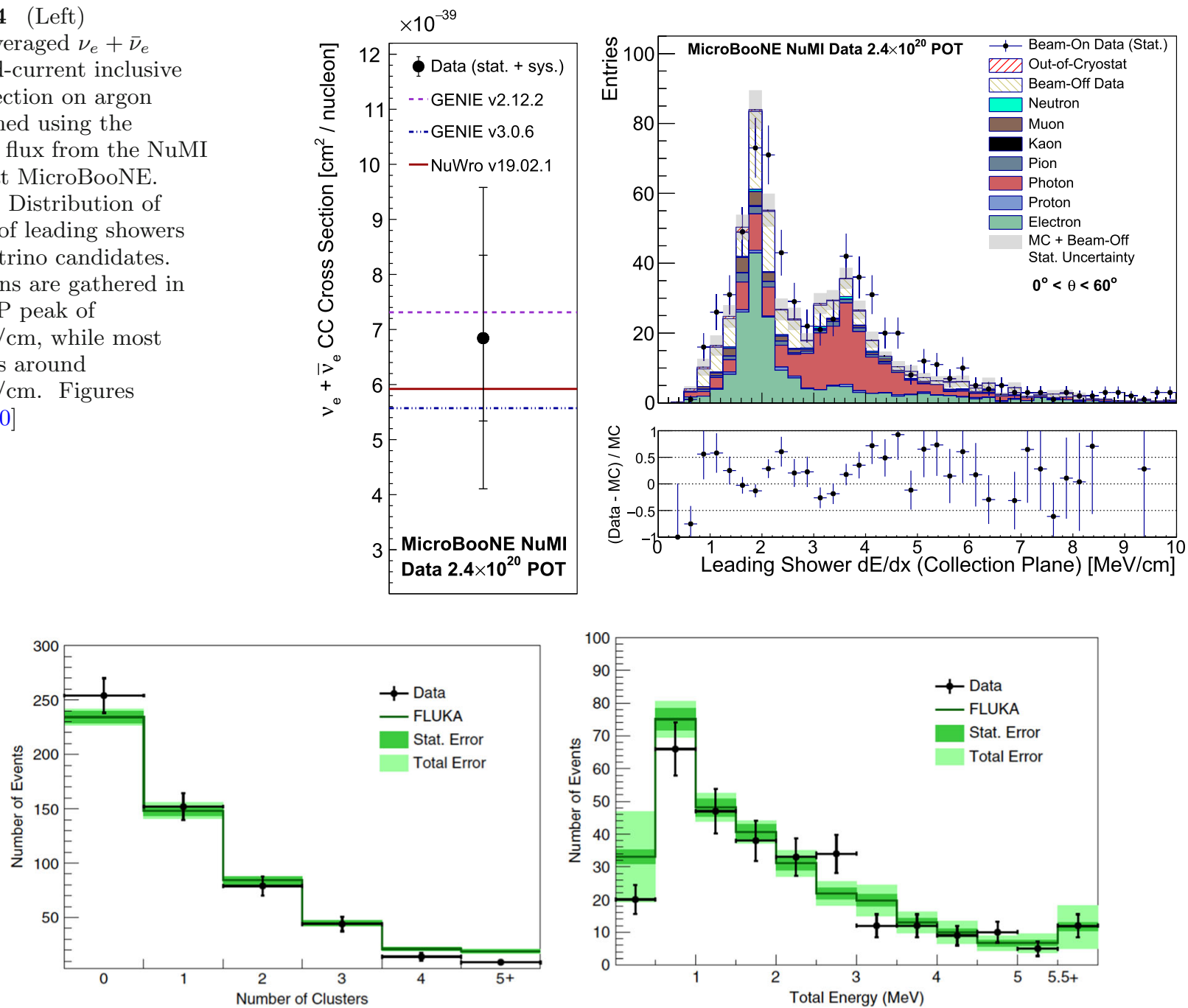

Fig. 15 (Left) Cluster multiplicity for neutrino data and FLUKA simulated events. (Right) Total signal reconstructed energy in an event for neutrino data and FLUKA simulated events. The simulation is normalized to the number of neutrino data events. Figure from [58]

previously measured on argon such as those associated with Transverse Kinematic Imbalance [53-56].

Extending the LArTPC's range of physics sensitivity down to the sub-MeV level is crucial for future studies of supernova, solar, and beam neutrino interactions. Furthermore, including charge deposits from deexcitation photons in $\mathrm{GeV}$ accelerator neutrino events in LArTPCs is expected to improve neutrino energy resolution by about 25\% [57]. ArgoNeuT has developed new techniques for low-energy LArTPC reconstruction for this purpose [58]. The total number, reconstructed energies, and positions of isolated low-energy depositions in the TPC volume were compared to those from simulations using the FLUKA Monte Carlo generator [59-61]. These measured features are consistent with energy depositions from photons produced by de-excitation of the neutrino's target nucleus and by inelastic scattering of primary neutrons produced by neutrino-argon interactions (see Fig. 15). Similar studies of low-energy reconstruction are underway in MicroBooNE.

Studies of low-energy new physics scenarios, such as millicharged particles [62], light mediators [63], and inelastic scatterings with small splittings [64], could also invaluably profit from such low-energy reconstruction. A search for millicharged particles, a simple extension of the standard model, has been performed with the ArgoNeuT detector [65].

\subsection{New detectors}

As well as new analysis techniques, new detectors are under construction that will add to the landscape of neutrino-argon cross section measurements. In the next few years, SBND will collect data in the same beamline as MicroBooNE, but will collect an order of magnitude more interactions. Expected statistics at SBND are 1.5 million CC-inclusive events, as well as 12,000 electron neutrino interactions [5]. This huge increase in statis- 
tics will allow multi-dimensional cross section measurements of a range of exclusive final states, including rare final states such as those including kaons and hyperons. Additionally, the ICARUS detector will make measurements of two GeV-scale neutrino beams over the next few years (the BNB on-axis and the NuMI beam off-axis), which will also include approximately 10,000 electron neutrino interactions in the NuMI dataset [5]. Further in the future, the DUNE near detector complex will include a liquid argon detector and a gaseousargon detector in a wide-band beam peaked at $4 \mathrm{GeV}$, expected to collect 30 million and 1.6 million $\nu_{\mu}$ CCinclusive events respectively per year in neutrino-mode beam running [66]. These data sets will be crucial to reach the precise understanding of neutrino-argon interactions needed for DUNE's oscillation program.

\section{Conclusions}

This article has summarized existing measurements from the ArgoNeuT and MicroBooNE collaborations, demonstrating the potential for LArTPCs to provide new insight into neutrino-nucleus interactions. The capabilities of this detector technology to study finalstate particle properties spanning a wide kinematic phase space with exquisite resolution opens up new avenues of inquiry, while the use of a nuclear target like argon requires improvements in modeling and analysis techniques to make precise measurements. Future highstatistics data samples collected by the SBN program, and the DUNE Near Detector, will be able to expand on the measurement techniques described in this article and further exploit the LArTPC technology to make exciting measurements of neutrino-nucleus interactions with improved precision.

Acknowledgements Work supported by the Fermi National Accelerator Laboratory, managed and operated by Fermi Research Alliance, LLC under Contract no. DEAC02-07CH11359 with the US Department of Energy. The US Government retains and the publisher, by accepting the article for publication, acknowledges that the US Government retains a non-exclusive, paid-up, irrevocable, worldwide license to publish or reproduce the published form of this manuscript, or allow others to do so, for US Government purposes.

\section{Author contribution statement}

All authors contributed equally to writing this manuscript. The reviews of ArgoNeuT measurements were written largely by OP, MS, and TY, and the reviews of MicroBooNE measurements were written largely by $\mathrm{KD}, \mathrm{APF}$, and $\mathrm{EG}$. KD was the main coordinator of the manuscript writing.
Open Access This article is licensed under a Creative Commons Attribution 4.0 International License, which permits use, sharing, adaptation, distribution and reproduction in any medium or format, as long as you give appropriate credit to the original author(s) and the source, provide a link to the Creative Commons licence, and indicate if changes were made. The images or other third party material in this article are included in the article's Creative Commons licence, unless indicated otherwise in a credit line to the material. If material is not included in the article's Creative Commons licence and your intended use is not permitted by statutory regulation or exceeds the permitted use, you will need to obtain permission directly from the copyright holder. To view a copy of this licence, visit http://creativecomm ons.org/licenses/by/4.0/.

\section{References}

1. C. Wilkinson et al., Testing charged current quasielastic and multinucleon interaction models in the NEUT neutrino interaction generator with published datasets from the MiniBooNE and MINER $\nu \mathrm{A}$ experiments. Phys. Rev. D 93(7), 072010 (2016). https://doi. org/10.1103/PhysRevD.93.072010 arXiv:1601.05592 [hep-ex]

2. L. Alvarez-Ruso et al., NuSTEC White Paper: status and challenges of neutrino-nucleus scattering. Prog. Part. Nucl. Phys. 100, 1-68 (2018). https://doi.org/10. 1016/j.ppnp.2018.01.006 arXiv:1706.03621 [hep-ph]

3. A. Lovato et al., Ab initio study of $\left(\nu_{l}, l^{-}\right)$and $\left(\bar{\nu}_{l}\right.$, $\left.l^{+}\right)$inclusive scattering in $12 \mathrm{C}$ : confronting the MiniBooNE and T2K CCQE data. Phys. Rev. X 10(3), 031068 (2020). https://doi.org/10.1103/PhysRevX.10. 031068 arXiv:2003.07710 [nucl-th]

4. B. Abi et al., Long-baseline neutrino oscillation physics potential of the DUNE experiment. Eur. Phys. J. C 80(10), 978 (2020). https://doi.org/10.1140/epjc/ s10052-020-08456-z

5. P.A.N. Machado, O. Palamara, D.W. Schmitz, The short-baseline neutrino program at Fermilab. Annu. Rev. Nucl. Part. Sci. 69, 363-387 (2019). https://doi. org/10.1146/annurev-nucl-101917-020949

6. C. Andreopoulos et al., The GENIE neutrino Monte Carlo generator. Nucl. Instrum. Methods A 614, 87104 (2010). https://doi.org/10.1016/j.nima.2009.12.009 arXiv:0905.2517 [hep-ph]

7. C. Andreopoulos et al., The GENIE neutrino Monte Carlo generator: physics and user manual (2015). arXiv:1510.05494 [hep-ph]

8. J. Tena-Vidal, GENIE Collaboration et al., Neutrinonucleon cross-section model tuning in GENIE v3. Phys. Rev. D 104(7), 072009 (2021). https://doi.org/10.1103/ PhysRevD.104.072009

9. T. Golan, C. Juszczak, J.T. Sobczyk, Effects of final-state interactions in neutrino-nucleus interactions. Phys. Rev. C 86(1), 015505 (2012). https://doi.org/10. 1103/PhysRevC.86.015505

10. O. Buss et al., Transport-theoretical description of nuclear reactions. Phys. Rep. 512(1), 1-124 (2012). ISSN:0370-1573. https://doi.org/10.1016/j.physrep. 2011.12.001 
11. Y. Hayato, A neutrino interaction simulation program library NEUT. Acta Phys. Pol. B 40, 2477 (2009)

12. R. Acciarri et al., Summary of the second workshop on liquid argon time projection chamber research and development in the United States. JINST 10(07), T07006 (2015). https://doi.org/10.1088/1748-0221/10/ 07/T07006 arXiv:1504.05608 [physics.ins-det]

13. T. Yang, Calibration of calorimetric measurement in a liquid argon time projection chamber. Instruments $\mathbf{5}(1)$, 2 (2020). https://doi.org/10.3390/instruments5010002 arXiv:2012.01319 [physics.ins-det]

14. C. Anderson et al., The ArgoNeuT detector in the NuMI low-energy beam line at Fermilab. JINST 7, P10019 (2012). https://doi.org/10.1088/1748-0221/7/ 10/P10019 arXiv:1205.6747 [physics.ins-det]

15. R. Acciarri et al., Design and construction of the MicroBooNE detector. JINST 12(02), P02017 (2017). https://doi.org/10.1088/1748-0221/12/02/P02017 arXiv:1612.05824 [physics.ins-det]

16. P. Adamson et al., The NuMI neutrino beam. Nucl. Instrum. Methods Phys. Res. Sect. A Accel. Spectrom. Detect. Assoc. Equip. 806, 279-306 (2016). ISSN:01689002. https://doi.org/10.1016/j.nima.2015.08.063

17. P. Adamson et al., Neutrino and antineutrino inclusive charged-current cross section measurements with the MINOS near detector. Phys. Rev. D $\mathbf{8 1}$, 072002 (2010). https://doi.org/10.1103/PhysRevD.81. 072002 arXiv:0910.2201 [hep-ex]

18. Aguilar-Arevalo et al., Neutrino flux prediction at MiniBooNE. Phys. Rev. D 79(7), 072002 (2009). https://doi. org/10.1103/PhysRevD.79.072002

19. C. Adams et al., Design and construction of the MicroBooNE Cosmic Ray Tagger system. J. Instrum. 14(04), P04004 (2019). https://doi.org/10.1088/1748-0221/14/ 04/p04004

20. P. Abratenko, MicroBooNE Collaboration et al., Measurement of the flux-averaged inclusive charged-current electron neutrino and antineutrino cross section on argon using the NuMI beam and the MicroBooNE detector. Phys. Rev. D 104, 052002 (2021). https://doi. org/10.1103/PhysRevD.104.052002

21. C. Anderson et al., First measurements of inclusive muon neutrino charged current differential cross sections on argon. Phys. Rev. Lett. 108(16), 161802 (2012). https://doi.org/10.1103/PhysRevLett. 108.161802 arXiv:1111.0103 [hep-ex]

22. R. Acciarri et al., Measurements of inclusive muon neutrino and antineutrino charged current differential cross sections on argon in the NuMI antineutrino beam. Phys. Rev. D 89(11), 112003 (2014). https://doi.org/10.1103/ PhysRevD.89.112003 arXiv:1404.4809 [hep-ex]

23. P. Abratenko et al., First measurement of inclusive muon neutrino charged current differential cross sections on argon at $E_{\nu} \sim 0.8 \mathrm{GeV}$ with the MicroBooNE detector. Phys. Rev. Lett. 123(13), 131801 (2019). https:// doi.org/10.1103/PhysRevLett.123.131801

24. P. Abratenko et al., Determination of muon momentum in the MicroBooNE LArTPC using an improved model of multiple Coulomb scattering. J. Instrum. 12(10), P10010 (2017). https://doi.org/10.1088/1748-0221/12/ $10 /$ p10010

25. P. Abratenko et al., Measurement of differential cross sections for $\nu_{\mu}$-Ar charged-current interactions with pro- tons and no pions in the final state with the MicroBooNE detector. Phys. Rev. D 102(11), 112013 (2020). https://doi.org/10.1103/PhysRevD.102.112013

26. C. Adams et al., Ionization electron signal processing in single phase LArTPCs. Part I. Algorithm description and quantitative evaluation with MicroBooNE simulation. J. Instrum. 13(07), P07006 (2018). https://doi. org/10.1088/1748-0221/13/07/p07006

27. C. Adams et al., Ionization electron signal processing in single phase LArTPCs. Part II. Data/simulation comparison and performance in MicroBooNE. J. Instrum. 13(07), P07007 (2018). https://doi.org/10. 1088/1748-0221/13/07/p07007

28. P. Abratenko et al., Measurement of space charge effects in the MicroBooNE LArTPC using cosmic muons. J. Instrum. 15(12), P12037 (2020). https://doi.org/10. 1088/1748-0221/15/12/p12037

29. C. Adams et al., A method to determine the electric field of liquid argon time projection chambers using a UV laser system and its application in Micro-BooNE. J. Instrum. 15(07), P07010 (2020). https://doi.org/10. 1088/1748-0221/15/07/p07010

30. K. Abe et al., Constraint on the matter-antimatter symmetry-violating phase in neutrino oscillations. Nature 580(7803), 339-344 (2020). https://doi.org/10. 1038/s41586-020-2177-0

31. A.A. Aguilar-Arevalo et al., Updated MiniBooNE neutrino oscillation results with increased data and new background studies. Phys. Rev. D 103(5), 052002 (2021). https://doi.org/10.1103/PhysRevD.103.052002

32. O. Palamara, Exclusive muon neutrino charged current pion-less topologies. ArgoNeuT results and future prospects in LAr TPC detectors, in Proceedings of the 10th International Workshop on Neutrino-Nucleus Interactions in Few-GeV Region (NuInt15), vol. 12 (2016), p. 010017. https://doi.org/10.7566/JPSCP.12. 010017

33. R. Acciarri et al., Detection of back-to-back proton pairs in charged-current neutrino interactions with the ArgoNeuT detector in the NuMI low energy beam line. Phys. Rev. D 90, 012008 (2014). https://doi.org/10. 1103/PhysRevD.90.012008 arXiv:1405.4261 [nucl-ex]

34. P. Abratenko et al., First measurement of differential charged current quasielasticlike $\nu_{\mu}$-argon scattering cross sections with the MicroBooNE detector. Phys. Rev. Lett. 125(20), 201803 (2020). https://doi.org/10. 1103/PhysRevLett.125.201803

35. R. Acciarri et al., First measurement of the cross section for $\nu_{\mu}$ and $\bar{\nu}_{\mu}$ induced single charged pion production on argon using ArgoNeuT. Phys. Rev. D 98(5), 052002 (2018). https://doi.org/10.1103/PhysRevD.98. 052002 arXiv:1804.10294 [hep-ex]

36. R. Acciarri et al., First measurement of neutrino and antineutrino coherent charged pion production on argon. Phys. Rev. Lett. 113(26), 261801 (2014). https://doi.org/10.1103/PhysRevLett.113.261801. arXiv:1408.0598 [hep-ex] [Erratum: Phys. Rev. Lett. 114, 039901 (2015)]

37. D. Rein, L.M. Sehgal, Coherent pi0 production in neutrino reactions. Nucl. Phys. B 223, 29-44 (1983). https://doi.org/10.1016/0550-3213(83)90090-1

38. H.J. Grabosch et al., Coherent pion production in neutrino and anti-neutrino interactions on nuclei of heavy 
Freon molecules. Z. Phys. C 31, 203 (1986). https://doi. org/10.1007/BF01479528

39. P. Vilain et al., Coherent single charged pion production by neutrinos. Phys. Lett. B 313, 267-275 (1993). https://doi.org/10.1016/0370-2693(93)91223-A

40. A. Higuera et al., Measurement of coherent production of $\pi^{ \pm}$in neutrino and antineutrino beams on carbon from $\mathrm{E}_{\nu}$ of $1: 5$ to $20 \mathrm{GeV}$. Phys. Rev. Lett. 113(26), 261802 (2014). https://doi.org/10.1103/PhysRevLett. 113.261802 arXiv:1409.3835 [hep-ex]

41. R. Acciarri et al., Measurement of $\nu_{\mu}$ and $\bar{\nu}_{\mu}$ neutral current $\pi^{0} \rightarrow \gamma \gamma$ production in the ArgoNeuT detector. Phys. Rev. D 96(1), 012006 (2017). https://doi.org/10. 1103/PhysRevD.96.012006

42. C. Adams et al., First measurement of $\nu_{\mu}$ chargedcurrent $\pi^{0}$ production on argon with the MicroBooNE detector. Phys. Rev. D 99(9), 091102 (2019). https:// doi.org/10.1103/PhysRevD.99.091102

43. A.A. Aguilar-Arevalo et al., Measurement of $\nu_{\mu}$-induced charged-current neutral pion production cross sections on mineral oil at $E_{\nu} \forall 0.5-2.0 \mathrm{GeV}$. Phys. Rev. D 83(5), 052009 (2011). https://doi.org/10.1103/PhysRevD.83. 052009

44. S.J. Barish et al., Study of neutrino interactions in hydrogen and deuterium. II. Inelastic charged-current reactions. Phys. Rev. D 19(9), 2521-2542 (1979). https://doi.org/10.1103/PhysRevD.19.2521

45. D. Rein, L.M. Sehgal, Neutrino-excitation of baryon resonances and single pion production. Ann. Phys. 133(1), 79-153 (1981). ISSN:0003-4916. https://doi. org/10.1016/0003-4916(81)90242-6

46. Ch. Berger, L.M. Sehgal, Partially conserved axial vector current and coherent pion production by low energy neutrinos. Phys. Rev. D 79(5), 053003 (2009). https:// doi.org/10.1103/PhysRevD.79.053003

47. M.A. Acero et al., New constraints on oscillation parameters from $\nu_{e}$ appearance and $\nu_{\mu}$ disappearance in the NOvA experiment. Phys. Rev. D 98, 032012 (2018). https://doi.org/10.1103/PhysRevD.98. 032012 arXiv:1806.00096 [hep-ex]

48. K. Abe et al., Hyper-Kamiokande design report (2018). arXiv:1805.04163 [physics.ins-det]

49. Aguilar-Arevalo et al., Significant excess of electronlike events in the mini-BooNE short-baseline neutrino experiment. Phys. Rev. Lett. 121(22), 221801 (2018). https://doi.org/10.1103/PhysRevLett.121.221801

50. M. Day, K.S. McFarland, Differences in quasi-elastic cross-sections of muon and electron neutrinos. Phys. Rev. D 86, 053003 (2012). https://doi.org/10.1103/ PhysRevD.86.053003 arXiv:1206.6745 [hep-ph]

51. A. Nikolakopoulos et al., Electron versus muon neutrino induced cross sections in charged current quasielastic processes. Phys. Rev. Lett. 123(5), 052501 (2019). https://doi.org/10.1103/PhysRevLett.123.052501

52. R. Acciarri et al., First measurement of electron neutrino scattering cross section on argon. Phys. Rev. D 102(1), 011101 (2020). https://doi.org/10.1103/ PhysRevD.102.011101 arXiv:2004.01956 [hep-ex]

53. X.-G. Lu et al., Measurement of nuclear effects in neutrino interactions with minimal dependence on neutrino energy. Phys. Rev. C 94(1), 015503 (2016). https://doi. org/10.1103/PhysRevC.94.015503
54. X. Lu, J.T. Sobczyk, Identification of nuclear effects in neutrino and antineutrino interactions on nuclei using generalized final-state correlations. Phys. Rev. C 99(5), 055504 (2019). https://doi.org/10.1103/PhysRevC.99. 055504

55. A.P. Furmanski, J.T. Sobczyk, Neutrino energy reconstruction from one-muon and one-proton events. Phys. Rev. C 95(6), 065501 (2017). https://doi.org/10.1103/ PhysRevC.95.065501

56. D. Coplowe et al., Probing nuclear effects with neutrinoinduced charged-current neutral pion production. Phys. Rev. D 102(7), 072007 (2020). https://doi.org/10.1103/ PhysRevD.102.072007

57. A. Friedland, S.W. Li, Understanding the energy resolution of liquid argon neutrino detectors. Phys. Rev. D 99, 036009 (2019). https://doi.org/10.1103/PhysRevD. 99.036009 arXiv:1811.06159 [hep-ph]

58. R. Acciarri et al., Demonstration of MeV-scale physics in liquid argon time projection chambers using ArgoNeuT. Phys. Rev. D 99, 012002 (2019). https://doi.org/10.1103/PhysRevD.99.012002 arXiv:1810.06502 [hep-ex]

59. A. Ferrari et al., FLUKA: a multi-particle transport code (Program version 2005). Technical report. No. CERN-2005-010, No. SLAC-R- 773, and No. INFN-TC05-11. 2005

60. G. Battistoni et al., Overview of the FLUKA code. Ann. Nucl. Energy 82, 10 (2015)

61. G. Battistoni et al., A neutrino-nucleon interaction generator for the FLUKA Monte Carlo code, in CERNProceedings-2010-001. Proceedings of 12th International Conference on Nuclear Reaction Mechanisms, Varenna, Italy, 15-19 June 2009 (2010), pp. 387-394

62. G. Magill et al., Millicharged particles in neutrino experiments. Phys. Rev. Lett. 122(7), 071801 (2019). https://doi.org/10.1103/PhysRevLett.122.071801 arXiv:1806.03310 [hep-ph]

63. S.N. Gninenko, New limits on radiative sterile neutrino decays from a search for single photons in neutrino interactions. Phys. Lett. B 710, 86-90 (2012). https:// doi.org/10.1016/j.physletb.2012.02.071 arXiv:1201.5194 [hep-ph]

64. E. Bertuzzo et al., Neutrino masses and mixings dynamically generated by a light dark sector. Phys. Lett. B 791, 210-214 (2019). https://doi.org/10.1016/ j.physletb.2019.02.023 arXiv:1808.02500 [hep-ph]

65. R. Acciarri et al., Improved limits on millicharged particles using the ArgoNeuT experiment at Fermilab. Phys. Rev. Lett. 124, 131801 (2020). https://doi.org/10.1103/ PhysRevLett.124.131801 arXiv:1911.07996 [hep-ex]

66. A. A. Abud, On behalf of the DUNE Collaboration et al., Deep underground neutrino experiment (DUNE) near detector conceptual design report. Instruments 5(4), 31 (2021). https://doi.org/10.3390/ instruments5040031 